\title{
Manifold Surface Reconstruction of an Environment from Sparse Structure-from-Motion Data
}

\author{
Maxime LHUILLIER and Shuda YU \\ Institut Pascal (ex. LASMEA), UMR 6602, CNRS/UBP/IFMA \\ Campus Universitaire des Cézeaux, \\ 63171 Aubière Cedex, France. \\ Email: Maxime.Lhuillier [at] free.fr \\ Tel: $+33(0) 473407593$ \\ Fax: +33(0)473407262
}

The reference of this paper is: Maxime Lhuillier and Shuda $\mathrm{Yu}$, Manifold Surface Reconstruction of an Environment from Sparse Structure-from-Motion Data, Computer Vision and Image Understanding, 117(11):1628-1644, 2013

This is the preprint version of the paper. The published version (DOI 10.1016/j.cviu.2013.08.002) is available at

http://www.sciencedirect. com/science/journal/10773142/117/11

\section{Copyright (C)2013 Elsevier}




\title{
Manifold Surface Reconstruction of an Environment from Sparse Structure-from-Motion Data
}

\author{
Maxime LHUILLIER and Shuda YU \\ Institut Pascal, UMR 6602, CNRS/UBP/IFMA, Aubière, France
}

\begin{abstract}
The majority of methods for the automatic surface reconstruction of an environment from an image sequence have two steps: Structure-from-Motion and dense stereo. From the computational standpoint, it would be interesting to avoid dense stereo and to generate a surface directly from the sparse cloud of 3D points and their visibility information provided by Structure-fromMotion. The previous attempts to solve this problem are currently very limited: the surface is non-manifold or has zero genus, the experiments are done on small scenes or objects using a few dozens of images. Our solution does not have these limitations. Furthermore, we experiment with hand-held or helmet-held catadioptric cameras moving in a city and generate 3D models such that the camera trajectory can be longer than one kilometer.
\end{abstract}

Keywords: 2-Manifold Reconstruction, 3D Delaunay Triangulation, Steiner Vertices, Complexity Analysis, Sparse Point Cloud, Structure-from-Motion.

\section{Introduction}

The topic of the paper is the reconstruction of a manifold surface using a sparse method. The definition and the importance of the manifold property are detailed in Sec. 1.1. Sec. 1.2 explains the motivations for a sparse method, i.e. a method which reconstructs a surface directly from the sparse point cloud estimated by Structure-from-Motion (SfM). Sec. 1.3 compares our work to the previous sparse methods and Sec. 1.4 summarizes our contributions.

Remember that SfM is a necessary and preliminary step which estimates the successive poses and (sometimes) intrinsic parameters of the camera from an image sequence. The sparsity comes from the fact that the SfM calculations are done for interest points, which have uneven distributions and low 
densities in the images (about 1 pixel over 200-300 is reconstructed in our experiments using Harris points [15]).

The sparse methods contrast with the predominant dense methods. There are two main cases of dense methods which estimate a manifold:

1. a surface evolves in $3 \mathrm{D}$ such that it minimizes a photo-consistency cost function for all image pixels [11, 17, 18, 31]

2. a surface reconstruction method $[41,6,21]$ is applied on a dense point cloud obtained by a dense stereo method [12, 22].

Combinations of both cases are possible (e.g. [23]) and an exhaustive list of references for dense methods is outside the paper scope. By contrast to the dense methods, the sparse methods are less popular and are a minority [10, $19,27,29,32,36,38]$ in the bibliography.

\subsection{Why a 2-Manifold?}

In a 2-manifold, i.e. a 2D topological manifold surface, every point of the surface has a surface neighborhood which is homeomorphic to a disk [5]. In short, a 2-manifold is parametrized by two real parameters. In the discrete case, a 2-manifold is usually defined by a list of triangles such that every triangle is exactly connected by its three edges to three other triangles.

The manifold property is required to enforce smoothness constraints on the computed surface. Indeed, the continuous differential operators of normal and curvature are extended to the discrete case thanks to this property $[28,5]$, and then we can use them to enforce smoothness constraints on the triangle list as in dense stereo [17]. More generally, a lot of Computer Graphic algorithms are not applicable if the triangle list is not a 2-manifold [5]. In our work, the manifold property is used to constrain a surface interpolating the sparse SfM point cloud and to improve surface denoising.

\subsection{Why a Sparse Method?}

There are several reasons to estimate a surface directly from the sparse SfM cloud. First, it would be ideal for both time and space complexities. This is interesting for obtaining compact models of large and complete environments like cities or for an implementation in a small embedded hardware. Second, it could be used for navigation pre-visualization [8], for initialization of dense stereo methods (e.g. [17, 18]), or for autonomous navigation [7]. Last, the accuracy of a point in SfM cloud is expected to be better than that

of a point in a dense stereo cloud, thanks to the SfM machinery [16] involving interest point detection and bundle adjustment. 

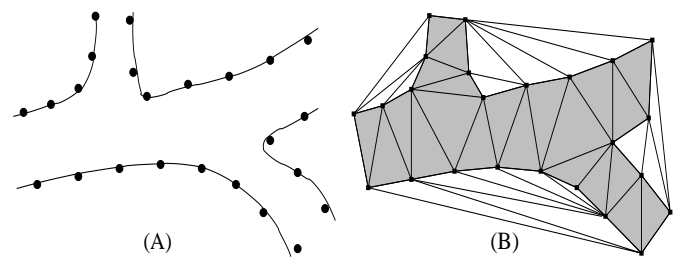

Figure 1: Delaunay-based reconstruction in the $2 \mathrm{~d}$ case (surfaces, tetrahedra and triangles are replaced by curves, triangles and edges, respectively). (A) curves to be reconstructed and points which sample the curves. (B) the Delaunay triangulation of the points. Note that the inside of [4] is the gray region, but our inside (Sec. 2) is the white region.

\subsection{Previous Works}

Methods [10, 19, 27, 29, 32, 36, 38] using our input (sparse SfM data) start by establishing potential adjacency relationships between the input points, which are selected latter to build a surface. These methods can be classified by examining the data structures encoding the potential adjacencies: $[10,32$, 27, 38] use one 3D Delaunay triangulation (Sec. 1.3.2) and [29, 36, 19] use 2D Delaunay triangulations (Sec. 1.3.3). The SfM data also includes visibility constraints, i.e. line segments (rays) which should not intersect the target surface, except at a segment end. A ray links a point to one of the view points used by SfM to reconstruct the point.

\subsubsection{Sculpting in a 3D Delaunay without Rays}

The sculpting method in [4] is closely related to our method, although it does not use rays. It partitions $\mathbb{R}^{3}$ into tetrahedra of the 3D Delaunay triangulation of the input points. The tetrahedra are labeled as inside or outside, and the surface is the list of triangles which bound the inside region (according to [2] and Fig. 1, a "good" surface can be obtained by such a segmentation). Every tetrahedron is initialized inside; the remainder of $\mathbb{R}^{3}$ is outside. Then an inside tetrahedron is selected by a geometric criterion and becomes outside while the resulting surface is manifold and every Delaunay vertex is in an inside tetrahedron. This method requires that the input point cloud is denser that ours and does not contains bad points.

\subsubsection{Sculpting in a 3D Delaunay with Rays}

Methods [10, 22, 32, 27, 38, 39] use rays to label the tetrahedra of the 3D Delaunay triangulation: a tetrahedron is freespace if it is intersected by ray(s), otherwise it is matter. 

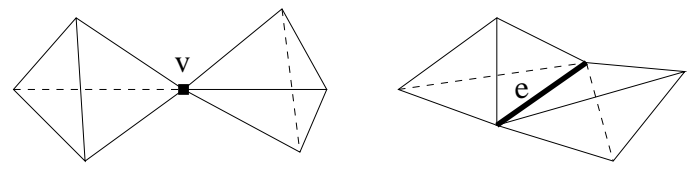

Figure 2: Surface singularities defined by two freespace tetrahedra in matter space.

In contrast to our method, [22, 32] do not enforce manifold constraint and [27] directly consider the surface as the list of triangles separating the freespace and matter tetrahedra. Then, the resulting surface can be nonmanifold. For example, the surface has a singularity at vertex $\mathbf{v}$ if all tetrahedra which have vertex $\mathbf{v}$ are matter, except two freespace tetrahedra $\Delta_{1}$ and $\Delta_{2}$ such that the intersection of $\Delta_{1}$ and $\Delta_{2}$ is exactly $\mathbf{v}$ (left of Fig. 2). Another singularity example is obtained if we replace "vertex $\mathbf{v}$ " by "edge e" in the previous example (right of Fig. 2). A post-processing [13] can be applied to obtain a 2-manifold by removing the singularities, but such an approach does not enforce the manifold constraint during the surface calculation and generates new vertices for every singular vertex.

Only [10] and our previous work [38, 39] provide a 2-manifold. In [10], a region growing procedure in the matter tetrahedra removes all surface singularities and reconstructs very simple scenes. In [38, 39], the region growing is in the freespace tetrahedra and deals with more complex topologies than those in $[10,4]$ : the estimated surface can be a torus with several handles. Our region growing is essentially a best first approach based on the number of ray intersections per tetrahedron ([10] does not use this information). In [39], artifacts called "spurious handles" and generated by [38] are removed while maintaining the manifold property. Here the largest handles are removed thanks to the tetrahedron labels (e.g. freespace). The previous removal methods [37, 42] remove the smallest handles without these labels.

\subsubsection{2d Delaunay-based Work}

Methods [29, 36, 19] use 2D Delaunay triangulations in images. In [19], the surface is not 2-manifold and the approach is applied to a small sequence of real images. In [29], the surface is a 2-manifold limited to a simple topology (sphere or plane). In [36], reconstructed edges are inserted in the constrained $2 \mathrm{D}$ triangulations, then the back-projected $2.5 \mathrm{~d}$ triangulations are merged by computing the union of the freespace defined by the $2.5 \mathrm{~d}$ triangulations. The resulting implicit surface is converted to manifold mesh by the marching cube method [26], which requires a thin-scale regular subdivision of space. 
However, an irregular space subdivision is better for large scale scene [22]. Compared to these methods, ours estimates a 2-manifold without topology limitation and without thin-scale regular space subdivision.

\subsection{Paper Contributions}

According to Sec. 1.3.2 and 1.3.3, our contributions over the other sparse methods are the following: we combine both 2-manifold and visibility constraints without limitation on the surface genus (i.e. the number of handles of the surface), and the experiments are done on larger image sequences of complete environments (hundreds/thousands of images in a few seconds/minutes). Furthermore, this paper is an extended version of our previous work [38, 39]. New material includes: highlight of our method with visibility optimization (Sec. 2), efficient tetrahedron-based manifold test (Sec. 2), discussion on bad SfM points (Sec. 3.6), complexity analysis (Sec. 5 and 6). Sec. 3 describes the overall method, then artifacts called "spurious handles" are removed by the methods in Sec. 4. Also there are new experiments in Sec. 7 about manifold constraint, varying densities of reconstructed and Steiner points, comparison with the Poisson surface reconstruction [21], larger still and video sequences. Note that we do not focus on the incremental surface reconstruction [40].

\section{Prerequisites on 2-Manifolds embedded in a 3D Delaunay}

Our algorithm needs prerequisites on 2-manifolds embedded in a 3D Delaunay triangulation. Here we introduce notations (Sec. 2.1), our optimization problem (Sec. 2.2), and the 2-manifold tests (Sec. 2.3).

\subsection{Notations and Tetrahedron Labeling}

Let $P$ be a set of reconstructed points. The 3D Delaunay triangulation of $P$ is a list $T$ of tetrahedra which meets the following conditions: the tetrahedra partition the convex hull of $P$, their vertex set is $P$, and the circumscribing sphere of every tetrahedron does not contain a vertex in its interior. A vertex/edge/triangle is a face of a tetrahedron in T. Assuming $L \subseteq T$, border $\delta L$ is the list of triangles which are included in exactly one tetrahedron of $L$. Let $F \subseteq T$ be the list of freespace tetrahedra, i.e. the tetrahedra intersected by ray(s). The tetrahedra which are not freespace

are matter. According to Sec. 1.3.2, $\delta F$ can be non-manifold. Here we introduce $O \subseteq F$, the list of outside tetrahedra, such that $\delta O$ is manifold. The tetrahedra which are not outside are inside. 
We also consider $T$ as a graph: a graph vertex is a tetrahedron, a graph edge is a triangle between two tetrahedra. A standard practice is to introduce infinite vertex $\mathbf{v}_{\infty}$ such that we define a virtual tetrahedron connecting $\delta T$ triangle and $\mathbf{v}_{\infty}$ [1]. The virtual tetrahedra do not exist in $3 \mathrm{D}$, but they are vertices of the graph. They make easier both implementation and paper clarity since every tetrahedron has exactly four neighbors in the graph.

Since $P$ is reconstructed in all directions around the view points to model complete environments, all rays are included in the convex hull of $P$. Then the virtual tetrahedra are labeled matter. Every matter tetrahedron is inside.

Note that $\delta F$ and $\delta O$ are closed surfaces; $\delta F$ cuts $\mathbb{R}^{3}$ into free-space $(F)$ and matter, $\delta O$ cuts $\mathbb{R}^{3}$ into outside $(O)$ and inside. Furthermore, $O \subseteq F$.

\subsection{Optimization Problem for 2-Manifold Extraction}

In short, the target surface $\delta O$ is a 2-manifold which should separate the matter and free-space tetrahedra "as much as possible". Let $r: F \rightarrow \mathbb{R}^{+*}$ a scalar and positive function. We extend $r$ to $O \subseteq F$ by $r(O)=\sum_{\Delta \in O} r(\Delta)$. We would like to estimate $O$ included in $F$ and maximizing $r(O)$ subject to the constraint that $\delta O$ is a 2-manifold. Intuitively (if $r=1$ ), a large $O$ in $F$ with a 2-manifold border $\delta O$ is a good solution. Now we give two remarks.

First, this optimization problem is difficult due to the manifold constraint. Then we solve it using a greedy algorithm: we add progressively free-space tetrahedra in $O$ as long as $\delta O$ remains a 2-manifold, then $r(O)$ increases and the final $\delta O$ is an approximation of the exact solution of our problem.

Second, several definitions of $r$ are possible. Here we optimize visibility by defining $r(\Delta)$ as the number of rays which intersect tetrahedron $\Delta$. Since

our paper focuses on manifold extraction and related complexity, we do not investigate on more sophisticated definitions of $r$ inspired by $[27,32,22,20]$.

\subsection{2-Manifold Tests}

Here we explain how to check that a surface $S$ (a list of triangles of $T$ ) is a 2 -manifold. This topic is somewhat technical but very important in practice for the computation time of our method.

Let $\mathbf{v}$ be a point in $S$. We say that $\mathbf{v}$ is regular if it has a neighborhood in $S$ which is topologically a disk. Otherwise $\mathbf{v}$ is singular. By definition (Sec. 1.1), $S$ is 2-manifold if all its points are regular. In our context where $S$ is a list of triangles of $T$, we only need to check that every vertex $\mathbf{v}$ of $S$ is regular using the following neighborhood of $\mathbf{v}$ : the list of the $S$ triangles which have vertex $\mathbf{v}[4]$. Now we describe three 2-manifold tests. 

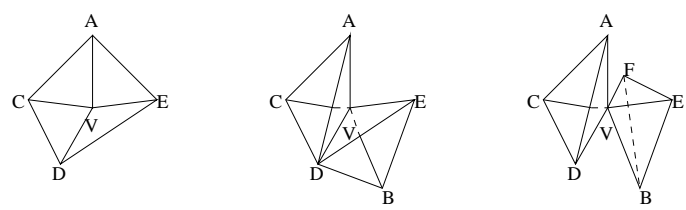

Figure 3: Regular and singular vertices. Left: $\mathbf{v}$ is regular since the edges opposite to $\mathbf{v}$ define a simple polygon acdea on the surface. Middle: $\mathbf{v}$ is singular since polygon dacdbed has multiple vertex $\mathbf{d}$. Right: $\mathbf{v}$ is singular since $\mathbf{a c d a}-\mathbf{b e f b}$ is not connected.

\subsubsection{Polygon-Based Vertex Test}

Vertex $\mathbf{v}$ is regular if and only if [4] the edges opposite to $\mathbf{v}$ in the triangles of $S$ having $\mathbf{v}$ as vertex form a simple polygon (Fig. 3). A simple polygon is topologically a circle, i.e. a list of segments which forms a closed path without self-intersection. This test was used in our previous work [38, 39].

\subsubsection{Graph-Based Vertex Test}

The test in Sec. 2.3.1 is adequate if the surface $S$ is implemented as an adjacency graph of triangles. This is not the case here since $S$ is embedded in a Delaunay $T$ implemented as an adjacency graph of tetrahedra.

So we introduce a faster test to check that $\mathbf{v}$ is regular. Let $g_{\mathbf{v}}$ be the graph of the tetrahedra incident to $\mathbf{v}\left(g_{\mathbf{v}}\right.$ is a sub-graph of $\left.T\right)$. Appendix A shows that the $\mathbf{v}$-opposite edges above form a simple polygon if and only if the inside tetrahedra of $g_{\mathbf{v}}$ are connected and the outside tetrahedra of $g_{\mathbf{v}}$ are connected (Fig. 4). Thus we check that $\mathbf{v}$ is regular thanks to a simple traversal of graph $g_{\mathbf{v}}$ where the edges between inside and outside are removed.

\subsubsection{Incremental Use of the Vertex Tests}

Now we explain how to use the 2-manifold tests in Sec. 2.3.1 and 2.3.2. In our context, $S=\delta O$ is estimated by a greedy method (Sec. 2.2). Assume that $\delta O$ is a 2-manifold. We add $A \subseteq F$ into $O$. Then we should check that (the new) $\delta O$ is still a 2-manifold. We collect in list $W$ all vertices of the tetrahedra in $A$ which are in $\delta O$. The new $\delta O$ is a 2-manifold if every vertex in $W$ is regular. Otherwise, it is not and $A$ is removed from $O$. The vertices of $\delta O$ which are not in $W$ do not need to be checked since the labels outside-inside of their incident tetrahedra do not change by adding $A$.

\subsubsection{Single Tetrahedron Test}

In the special case where list $A$ contains a single tetrahedron $\Delta$, there is a test based on [4], which is faster than those in Sec. 2.3.3. In this case, we 


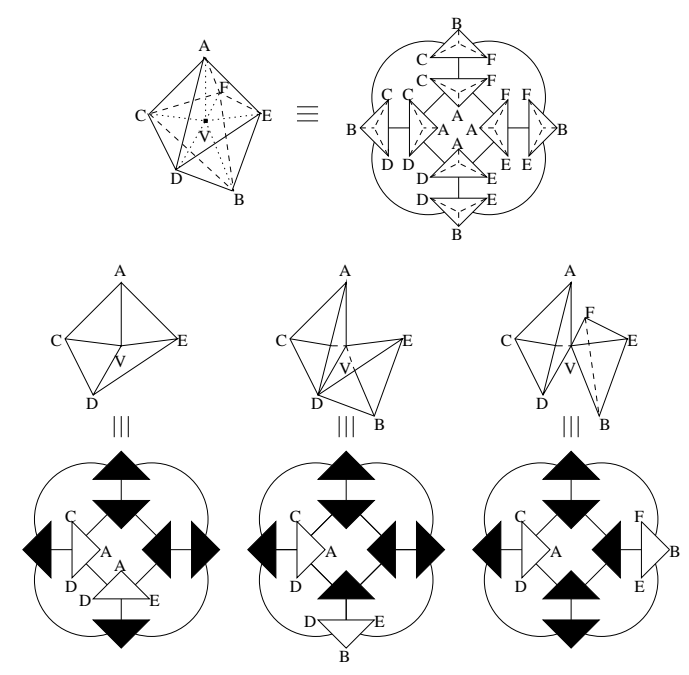

Figure 4: Relation between topology and graph. Top: 8 tetrahedra incident to $\mathbf{v}$ and their adjacency graph $g_{\mathbf{v}}$. Bottom: three cases of tetrahedra labeling (inside is black, outside is white). Middle: the resulting topology around $\mathbf{v}$. Middle and bottom: $\mathbf{v}$ is regular if and only if the inside tetrahedra are connected and the outside tetrahedra are connected.

first check a condition on $O$ and then add $\Delta$ to $O$ (if the condition is meet). This condition is detailed in Appendix B. It only requires to read once the lists of tetrahedra incident to the four $\Delta$-vertices.

\section{Surface Reconstruction Algorithm}

Every sub-section describes a step of our method, except the last one which discusses bad (false positive) SfM points.

\subsection{D Delaunay Triangulation $T$}

Assume that SfM estimates the geometry of the whole image sequence. The geometry includes the sparse cloud of points $\left\{\mathbf{p}_{i}\right\}$, camera locations $\left\{\mathbf{c}_{j}\right\}$ and rays defined by visibility lists $\left\{V_{i}\right\}$. List $V_{i}$ is the list of indices of images which reconstruct the $3 \mathrm{D}$ point $\mathbf{p}_{i}$, and a ray is a line segment linking $\mathbf{p}_{i}$ to $\mathbf{c}_{j}$ if $j \in V_{i}$. The size of $V_{i}$ is greater than 2 (2 is the theoretical minimum but it is insufficient for robustness).

Point $\mathbf{p}_{i}$ has poor accuracy if it is reconstructed in degenerate configuration [16]: if $\mathbf{p}_{i}$ and all $\mathbf{c}_{j}, j \in V_{i}$ are nearly collinear. This case occurs in part of the camera trajectory which is a straight line and if points reconstructed 
from this part are close to the straight line. Thus, the point cloud is filtered as in [9]: $\mathbf{p}_{i}$ is added in $T$ if and only if there is $\{j, k\} \subseteq V_{i}$ such that angle $\widehat{\mathbf{c}_{j} \mathbf{p}_{i} \mathbf{c}_{k}}$ meets $\epsilon \leq \widehat{\mathbf{c}_{j} \mathbf{p}_{i} \mathbf{c}_{k}} \leq \pi-\epsilon$ using threshold $\epsilon>0$.

\subsection{Ray Tracing}

We set $r=0$ and apply ray tracing to all rays. Since $T$ is a graph (Sec. 2.1), tracing a ray $\mathbf{c}_{j} \mathbf{p}_{i}$ is a walk in the graph, starting from a tetrahedron incident to $\mathbf{p}_{i}$, moving to another tetrahedron through the triangle intersected by the line segment $\mathbf{c}_{j} \mathbf{p}_{i}$, and stopping to the tetrahedron which contains $\mathbf{c}_{j}$ (the inverse walk is also possible). Ray $\mathbf{c}_{j} \mathbf{p}_{i}$ is traced if and only if $\mathbf{p}_{i}$ is a vertex of $T$. For every tetrahedron $\Delta$ intersected by a ray, $r(\Delta)$ is increased by 1 . We obtain function $r$ and list $F$ of the free-space tetrahedra.

\subsection{2-Manifold Extraction}

According to Section 2.2, we use a greedy algorithm to approximate the list $O$ which maximizes $r(O)$ such that $\delta O$ is a 2-manifold and $O \subseteq F$ : $O$ grows from $\emptyset$ by adding free-space tetrahedra such that $\delta O$ remains 2 manifold. The result of this step is the final $\delta O$. We use $r$ to define a priority for the free-space tetrahedra: the $\Delta \mathrm{s}$ with the largest $r(\Delta)$ are added in $O$ before others. Due to the manifold constraint, we also require that the selected $\Delta$ has (at least) one face in $\delta O$ to avoid that the greedy algorithm gets stuck too easily in a bad solution

The tetrahedra in the neighborhood of $O$ are stored in a heap (priority queue) for fast selection of the tetrahedron with the greatest $r$. Thus $O$ usually grows from the most confident free-space tetrahedra (with large $r$ ) to the less confident ones (with small $r$ ).

Algorithm 1 presents our growing method in $\mathrm{C}$ style. The inputs are the initial $O$, list $F$, list $Q_{0}$ of tetrahedra which includes the initial value of the heap, and function $r$. Here we use $O=\emptyset$ and $Q_{0}=\emptyset$. The output is $O$.

\subsection{Topology Extension}

The outside region $O$ computed in Sec. 3.3 has the ball topology since we add tetrahedra one-by-one [4]. This is problematic if the true outside does not have the ball topology, e.g. if the camera trajectory contains closed loop(s) around building(s). In the simplest case of one loop, the true outside has the toroid topology and the computed outside $O$ can not close the loop (as shown by Fig. 5). 

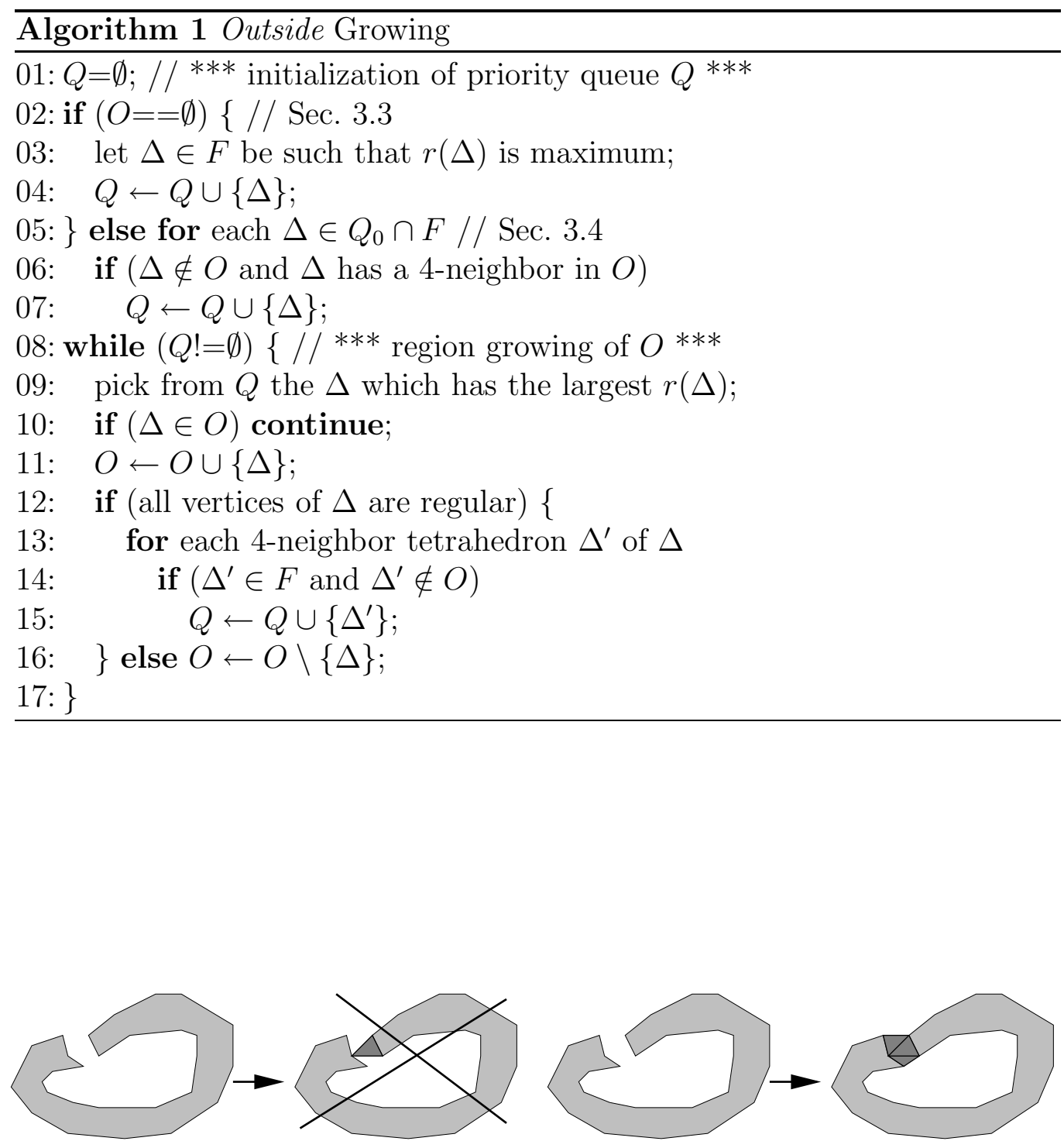

Figure 5: Close the loop. Left: adding one tetrahedron (dark) at once in $O$ (light) can not close the loop due to singular vertex. Right: adding several tetrahedra at once can do it. 
This problem is corrected as follows. Firstly, we find a vertex in $\delta O$ such that all inside tetrahedra incident to this vertex are free-space. Secondly, these tetrahedra are collected in list $A$. Thirdly, we try to add $A$ into $O$ using a 2-manifold test in Sec. 2.3. At last, if the test is successful, we use the region growing in Sec. 3.3 where $Q_{0}$ is the list of tetrahedra neighbors of $A$. In practice, we go through the list of $\delta O$ vertices several times to do this process.

\subsection{Post-Processing}

Although the surface $S$ provided by the previous steps is a 2-manifold $(S=\delta O)$, it has several weaknesses which are easily noticed during visualization. Now we examine these weaknesses and explain how to remove or reduce them using prior knowledge of the scene.

\subsubsection{Peak Removal}

A peak is a vertex $\mathbf{p}_{i}$ on $S$ such that the ring of its incident triangles in $S$ defines a solid angle $w$ which is too small to be physically plausible, i.e. $w<w_{0}$ where $w_{0}$ is a threshold. Let $L$ be the list of the tetrahedra in the acute side of the peak ring. $L$ is outside or inside. Now we reverse the $L$ label: inside becomes outside, and vice versa. The removal of peak $\mathbf{p}_{i}$ from $S$ is successful if all vertices of the $L$ tetrahedra remain regular. Otherwise, the label of $L$ is restored to its original value and we try to remove another peak. In practice, we go through the list of $S$ vertices several times to detect and remove peaks. Note that this step ignores the free-space/matter labeling.

\subsubsection{Surface Denoising}

The $S$ reconstruction noise is reduced thanks to a smoothing filter $\mathbf{p}^{\prime}=$ $\mathbf{p}+\Delta \mathbf{p}$ where $\mathbf{p}$ is a vertex of $S$ and $\Delta \mathbf{p}$ is a discrete Laplacian defined on $S$ vertices [35]. The smoothed $\mathbf{p}^{\prime}$ is stored in a distinct array of $\mathbf{p}$. We don't apply $\mathbf{p} \leftarrow \mathbf{p}^{\prime}$ to avoid the computation overhead due to vertex update in $T$.

\subsubsection{Sky Removal}

Up to now, $S$ is closed and contains triangles which correspond to the sky (assuming outdoor image sequence). These triangles should be removed since they do not approximate a real surface. They also complicate the visualization of the 3D model from a bird's-eye view. Firstly, the upward vertical direction $\mathbf{u}$ is robustly estimated assuming that the camera motion is (roughly) on a horizontal plane. Secondly, we consider open rectangles 
defined by the finite edge $\mathbf{c}_{i} \mathbf{c}_{i+1}$ and the two infinite edges (half lines) starting from $\mathbf{c}_{i}$ (or $\mathbf{c}_{i+1}$ ) with direction $\mathbf{u}$. A triangle of $S$ which is intersected by an open rectangle is a sky triangle and is removed from $S$. Now $S$ has hole in the sky. Lastly, the hole is increased by propagating its border from triangle to triangle while the angle between triangle normal (oriented from outside to inside) and $\mathbf{u}$ is less than threshold $\beta$.

\subsection{Bad (False Positive) SfM Points}

SfM can reconstruct bad point $\mathbf{p}$ due to repetitive texture or image noise. Now we discuss the consequences and give solutions for this problem. If $\mathbf{p}$ is in the true matter of the scene, there are tetrahedra that should be matter which are labeled free-space due to bad ray of $\mathbf{p}$. If these tetrahedra are in $O, \delta O$ can be corrupted, e.g. a wall with a spurious concavity. In practice, the risk of bad $\mathbf{p}$ is low thanks to the SfM machinery (bundle adjustment, RANSAC, robust matching, interest point detectors).

Nevertheless, our method reduces this risk and can remove spurious concavities. First, the risk is reduced thanks to the choice of $\epsilon$ in the point selection step (Sec. 3.1): the larger $\epsilon$, the more accurate points and their rays used by Ray Tracing (Sec. 3.2), the lower risk of spurious concavity. Second, the spurious concavities created by the manifold constraint in the growing steps (Sec. 3.3 and 3.4) can not be worse (larger) than those of the free-space in the matter. Indeed, a concavity is a list of outside tetrahedra which are included in free-space. Third, Appendix $\mathrm{C}$ explains how Peak Removal (Sec. 3.5.1) can remove spurious concavities.

\section{Removal of Spurious Handles}

Sec. 4.1 introduces spurious handles. Then two removal methods are presented in Sec. 4.2 and 4.3, which complete our methods in Sec. 3 (the removal methods are not presented in Sec. 3 for the paper clarity).

\subsection{Definition of Spurious Handles}

Topology Extension (Sec. 3.4) calculates a 2-manifold without genus constraint and improves our solution of the optimization problem (Sec. 2.2), but it has one drawback: it can generate spurious handles. Fig. 6 shows an example in a real case: the oblique handle on the left connects a small wall to the ground. This handle is spurious: it does not exist on the true scene surface and it should be removed while maintaining the manifold property. 


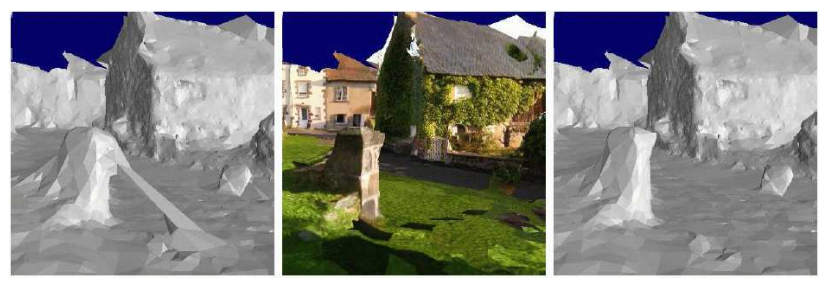

Figure 6: Spurious handle (left) and its removal (middle and right).

In the paper, we remove the handles which are both "visually critical" and due to "incomplete" outside growing in the free-space. "Visually critical" means that we ignore the handles that are too small to be easily noticeable by viewers (virtual pedestrians) located at all view points $\mathbf{c}_{j}$ reconstructed by SfM. "Incomplete" means that the handles only contains free-space tetrahedra that are inside and which should be forced to outside. We use these conditions to localize the spurious handles and to obtain a final 2-manifold which meets the visibility constraints provided by the rays. For the remainder of the paper, a "spurious handle that is visually critical and due to incomplete growing" is shortened to "spurious handle".

Our spurious handle removal methods use Steiner vertices, i.e. extra points in $T$ which are not in the original (SfM) input. The Steiner vertices do not have rays.

\subsection{Method 1: Reduce Size of Tetrahedra}

In [38], a simple method is used to reduce the risk of spurious handles. In the 3D Delaunay step (Sec. 3.1), Steiner points are added in $T$ such that the long tetrahedra potentially involved in spurious handles are split in smaller tetrahedra. The Steiner points are added in the critical region for visualization: the immediate neighborhood of the camera trajectory. We randomly add a fixed and small number of Steiner vertices at the neighborhood of every camera location $\mathbf{c}_{j}$. A neighborhood is a ball centered at $\mathbf{c}_{j}$ with radius defined as a multiple (e.g. 10) of $\operatorname{mean}_{j}|| \mathbf{c}_{j+1}-\mathbf{c}_{j} \|$.

\subsection{Method 2: Detect, Force and Repair}

Another removal method [39] is applied after "Topology Extension" and before "Post-Processing". Every sub-section describes a step of the method. 


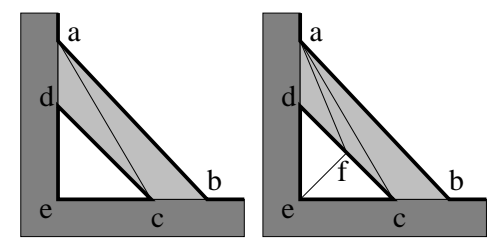

Figure 7: Spurious handle (left) and Edge Splitting (right) in the 2D case. Bold edges $=$ manifold edges, simple edges $=$ Delaunay edges, white region $=$ outside, light gray $=$ insidefreespace, dark gray $=$ inside-matter. Left: edges $\mathbf{a b}, \mathbf{c d}$, ac are visually critical. Right: Steiner point $\mathbf{f}$ is inserted on $\mathbf{c d}$, this splits triangle cde and adc.

\subsubsection{Detect Visually Critical Edges}

Let $e$ be an edge with finite vertices $\mathbf{a}_{e}$ and $\mathbf{b}_{e}$. Let $\alpha>0$ be a threshold. Edge $e$ is "visually critical" if

1. every tetrahedron including $e$ is free-space and

2. at least one inside tetrahedron includes $e$ and

3. there is a view point $\mathbf{c}_{j}$ such that angle $\widehat{\mathbf{a}_{e} \mathbf{c}_{j} \mathbf{b}_{e}}$ is greater than $\alpha$.

The first step is the calculation of all visually critical edges in list $L_{\alpha}$. A spurious handle has critical edges both on its border and its interior (Fig. 7).

The larger $\alpha$, the smaller size of $L_{\alpha}$ and also the greater lengths of the edges in $L_{\alpha}$. We use this to select a moderated number of handles and apply on every selected handle a processing, whose time computation per tetrahedron/vertex is greater than those in Sec. 3.3 and 3.4.

\subsubsection{Force Tetrahedra Outside}

This step needs a mesh operator called "Edge Splitting". It splits an edge of $T$ by adding a Steiner vertex on the edge, and every tetrahedron including the edge is also split in two tetrahedra whose labels (free-space, matter, inside, outside) are the same as the original tetrahedra. Thus, the resulting triangulation $T$ is not $100 \%$ Delaunay after this step, but our surface $\delta O$ which separates outside and inside tetrahedra is still a 2-manifold (the set of surface points is unchanged).

Now we split every $L_{\alpha}$ edge by a Steiner vertex on its middle (one example in Fig. 7). This changes the graph of tetrahedra and provides new tetrahedra for the next step, which is a local region growing of $O$ in $F$. For each vertex $\mathbf{v}_{i}$ on (split) critical edges, we define the list of tetrahedra $L_{i}$ incident to $\mathbf{v}_{i}$ which are free-space and inside. Then a first round of (Force,Repair) is applied to the list $L_{i}$. Function "Force" adds tetrahedra in $O$ without checking that $\delta O$ 
is a 2-manifold, and function "Repair" (Sec. 4.3.3) tries to grow $O$ such that $\delta O$ becomes a 2-manifold. If it fails, another round of (Force,Repair) is tried for every tetrahedron of $L_{i}$. Algorithm 2 presents our method in $\mathrm{C}$ style.

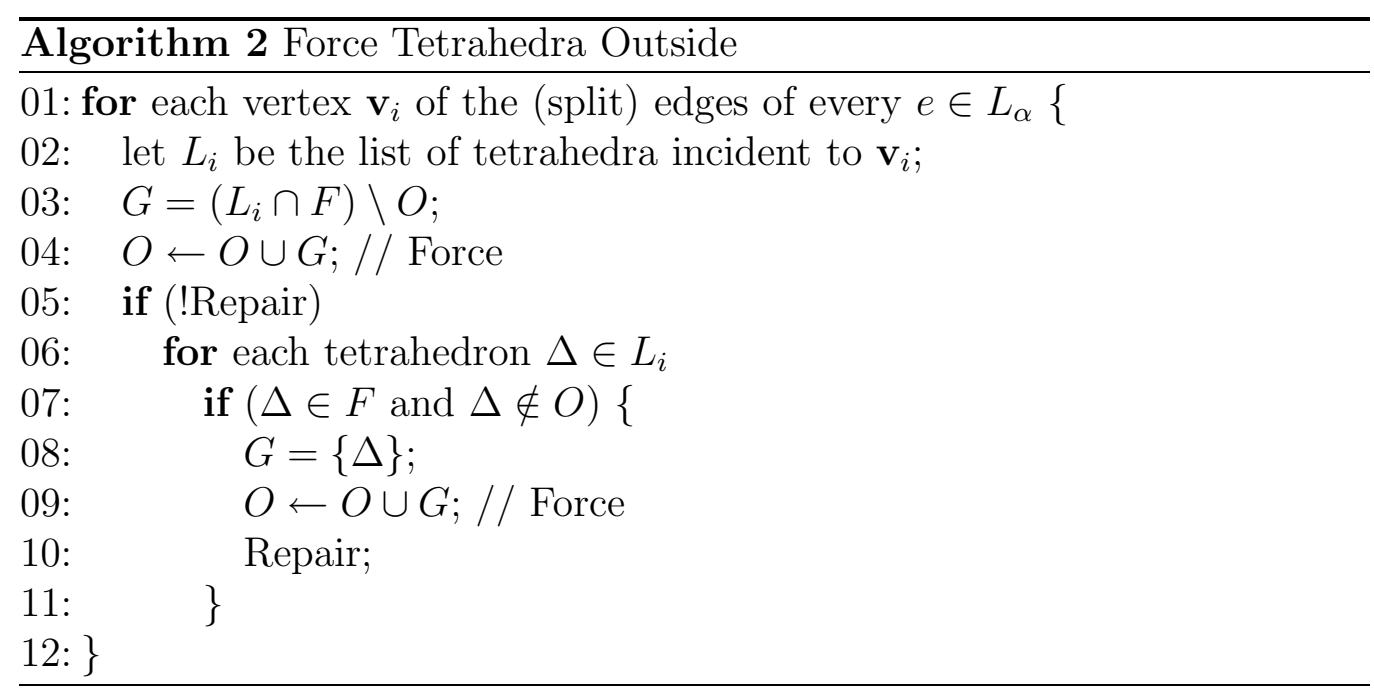

\subsubsection{Repair $\delta O$ by local growing of $O$}

The repair step is a local growing of $O$ in $F$ such that the number $n$ of singular vertices of surface $\delta O$ decreases and every regular vertex is maintained regular. At the beginning, $n>0$ is due to the addition of $G$ in $O$ (Force). Then a free-space and inside tetrahedron $\Delta$ is chosen in the neighborhood of $G$, and it is added to $O$. If $n$ increases, we remove $\Delta$ from $O$ and try another tetrahedron. We continue until every $\Delta$ candidate increases $n$. If the final $n$ is 0 , the local region growing succeeds. Otherwise it fails and $O$ is restored to its value before the Force step.

The inputs are $O, G$ and $g_{0}$ (an upper bound to limit the local growing complexity). The output is a boolean which asserts that Repair is successful or failed. Repair can also modify $O$. Algorithm 3 presents function Repair in $\mathrm{C}$ style. Note that this algorithm looks like the one in Sec. 3.3. The main differences are (1) the 2-manifold tests are replaced by non-increase tests of the number of singular vertices and (2) the process can fail.

\section{Starting the Complexity Analysis}

Here we evaluate the time complexities of the 2-manifold tests (Sec. 5.2) and region growing (Sec. 5.3) using notations and data structures in Sec. 5.1. 


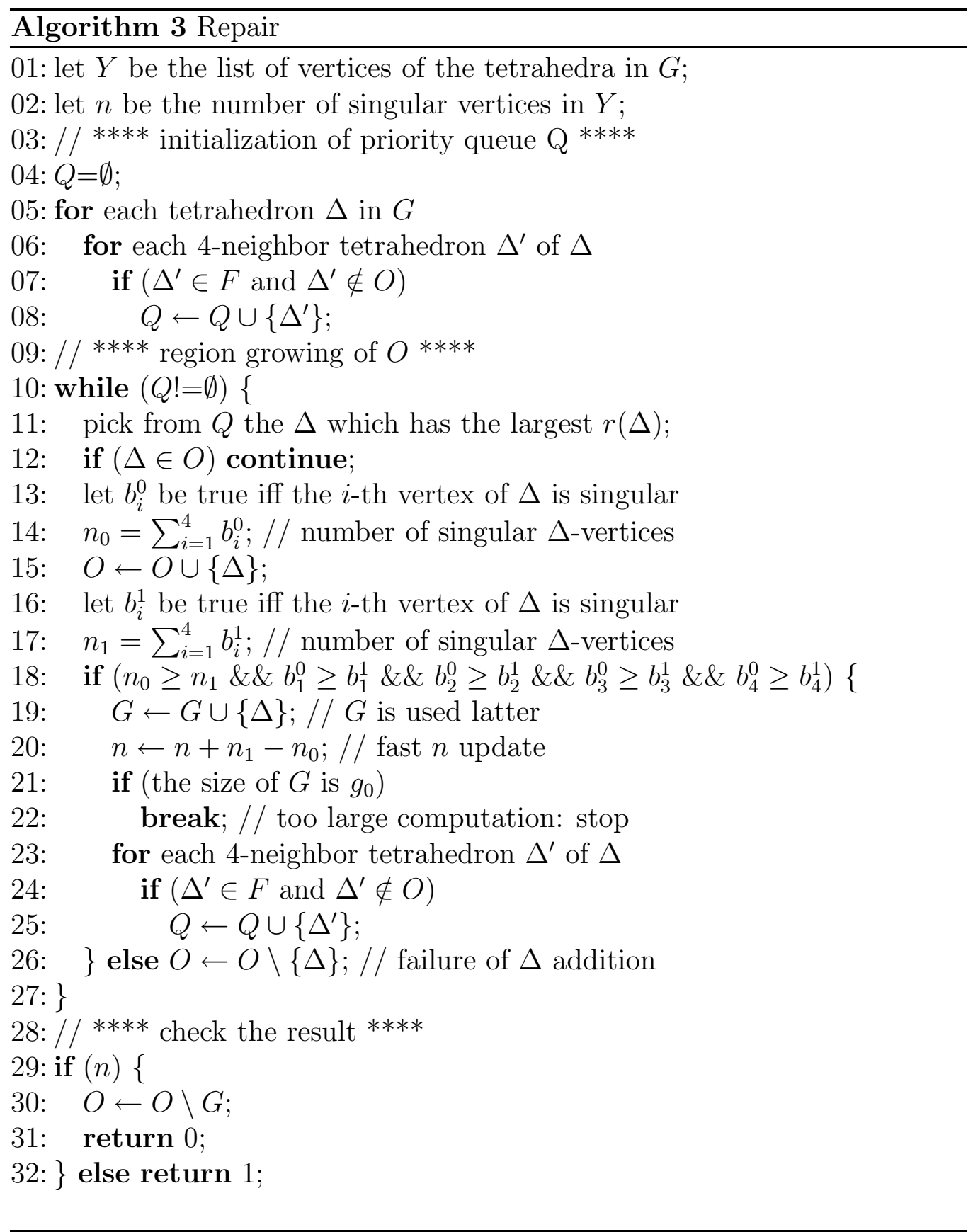


Then Sec. 5.4 discusses the worst case complexities of these steps and others. We use the standard "big o" notation $\mathcal{O} ; x$ is bounded if $x=\mathcal{O}(1)$.

\subsection{Notations and Data Structures}

The 3D Delaunay triangulation $T$ is the labeled graph defined in Sec. 2.1. We note $|L|$ the number of elements of list $L ;|T|$ is the number of tetrahedra. Let $v$ be the number of tetrahedra vertices. We assume that every tetrahedron $\Delta$ stores a label outside-inside, the number $r(\Delta)$ of intersected rays, the list of 4 vertices, and the list of 4 neighbor tetrahedra. The tetrahedra and their vertices are referenced by integers in the lists above. There is a table of vertices and a table of tetrahedra. Note that $\Delta \in F$ if and only if $r(\Delta)>0$. Let $d$ be the maximum vertex degree, i.e. the number of tetrahedra incident to every finite vertex (every vertex except $\mathbf{v}_{\infty}$ ) is less or equal to $d$.

\subsection{Complexity of the 2-Manifold Tests}

In Sec. 2.3.3, we add $A$ to $O$ if and only if $\delta O$ remains 2-manifold, i.e. if every vertex of $W$ is regular. According to Sec. 3.3 and 3.4, $A$ has a single tetrahedron or several tetrahedra sharing a surface vertex. This vertex can not be $\mathbf{v}_{\infty}$ since all $\mathbf{v}_{\infty}$-incident tetrahedra are matter and every surface vertex is incident to a free-space tetrahedron. Thus, $|A| \leq d$. Since $W$ is the list of the vertices of the tetrahedra in $A$, its complexity is $\mathcal{O}(d)$. Furthermore, the complexity to check that a vertex is regular using the Graph-Based Test in Sec. 2.3.2 is $\mathcal{O}(d)$. Thus the time complexity of the 2-manifold test for list $A$ is $\mathcal{O}\left(d^{2}\right)$. If $A$ has a single tetrahedron, both graph-based method (Sec. 2.3.2) and single tetrahedron method (Appendix B) are $\mathcal{O}(d)$.

\subsection{Complexity of One Region Growing}

Here we estimate the time complexity of Algorithm 1. Let $q_{0}$ be the number of tetrahedra in list $Q_{0}$. Let $g$ be the number of grown tetrahedra, i.e. the difference between the number of tetrahedra in the output $O$ and the number of tetrahedra in the input $O$ of this algorithm. A tetrahedron $\Delta$ is definitely added at most one time into $O$. In this case, at most four tetrahedra are added to $Q$. There is no other addition to $Q$, except at the initialization of $Q$ where there are at most $\mathcal{O}\left(q_{0}\right)$ additions to $Q$. Thus, the number of "while" iterations is $\mathcal{O}\left(g+q_{0}\right)$.

Now the time complexity of one iteration is estimated. Picking the best tetrahedron in heap $Q$ is $\mathcal{O}\left(\log \left(g+q_{0}\right)\right)$. The other instructions in one "while" iterations are $\mathcal{O}(d)$ due to the 2-manifold test (Sec. 5.2 using $|A|=1$ ). 
If we start from $O=\emptyset$, we should add the complexity of $Q$ initialization: the time complexity of one region growing is $\mathcal{O}(|T|+g(d+\log (g)))$. If we start from $O \neq \emptyset$, this time complexity is $\mathcal{O}\left(\left(g+q_{0}\right)\left(d+\log \left(g+q_{0}\right)\right)\right)$.

\subsection{Loose Results}

The number of tetrahedra is $|T|=\mathcal{O}\left(v^{2}\right)$ and the maximum vertex degree is $d=\mathcal{O}(v)$ since $T$ has $v$ vertices $[3,34]$; the worst case exists but it is

rare. Furthermore, a theoretical study starting from these numbers provides time complexities that are not tight enough to be interesting. Here are two examples. First, the time complexity in the worst case to trace a single ray is $\mathcal{O}\left(v^{2}\right)$. Indeed, one line segment can intersect all tetrahedra [34], but this is almost impossible in practice. A second example is the region growing which is $\mathcal{O}\left(v^{3}\right)$ in the worst case (use $d=\mathcal{O}(v)$ and $g+q_{0}=\mathcal{O}\left(v^{2}\right)$ in Sec. 5.3), but it is quite smaller in practice. So we will use neither $|T|=\mathcal{O}\left(v^{2}\right)$ nor $d=\mathcal{O}(v)$ in the complexity analysis below.

\section{Tight Time Complexity}

Sec. 6.1 introduces two new assumptions: the bounded density of the SfM points and the addition in $T$ of a Cartesian grid of Steiner vertices. Sec. 6.2 lists all our assumptions and Sec. 6.3 provides the worst case time complexities for the steps of our method.

\subsection{Additional Point Assumptions and their Consequences}

We assume that the density of reconstructed points in 3D is bounded: there are $p>0$ and $q>0$ such that every $p$-ball contains at most $q$ points. A $p$-ball is a ball with radius $p$. This could be justified as follows: (1) only interest points are reconstructed and (2) the scene surface has texture such that the interest points, which are detected due to grey-level 2D variations in their neighborhood, have a bounded density (e.g. no fractal-like texture).

We also use a bounding box of the reconstructed points, which is computed from the point coordinates. This box has Delaunay mesh $T$ with two kinds of vertices: the reconstructed vertices (Sec. 3.1) and Steiner vertices located at the corners of a Cartesian grid. The adding of Steiner vertices (i.e. extra points) is a standard method in Computational Geometry to generate meshes with good properties, e.g. to guarantee a linear-size 3D Delaunay triangulation with bounded vertex degree [3]. Every Steiner vertex has an 
empty visibility list. In practice, the number $s$ of Steiner vertices is quite smaller than the number $m$ of reconstructed vertices $(v=m+s \approx m)$.

Thanks to the bounded density of the SfM points and the Cartesian grid of Steiner vertices, Appendix D shows that the tetrahedron density is also bounded, i.e. there are $p^{\prime}>0$ and $q^{\prime}>0$ such that every $p^{\prime}$-ball intersects at most $q^{\prime}$ tetrahedra. The proof has two steps: first the tetrahedron diameter is bounded by the diagonal length $l$ of a grid voxel, second we use the bounded density of the points in the $p^{\prime}+l$-ball which includes all tetrahedra intersecting the $p^{\prime}$-ball. If the $p^{\prime}$-ball is centered at a finite vertex, we see that the maximum vertex degree $d$ is bounded.

\subsection{List of Properties and Assumptions}

This list is used in the complexity proofs.

H0: $T$ has $m$ reconstructed and $s$ Steiner vertices where $s<m$

H1: the tetrahedron density is bounded

H2: the maximum vertex degree $d$ is bounded

H3: the access to the 4 neighbors of a tetrahedron is $\mathcal{O}(1)$

$\mathrm{H} 4$ : the size of visibility list $V_{i}$ is bounded

H5: the distance between two consecutive view points is bounded.

H0-2 are in Sec. 6.1. H3 is in Sec. 5.1. In practice, the distance between the locations of two successive images is bounded (H5) and we assume that the track length (of interest point in successive images) is also bounded (H4).

\subsection{Worst Case Time Complexities}

Tab. 1 gives the time complexities of the steps of our method. Here we briefly summarize these results. The detailed proofs are in Appendix E.

Step "3D Delaunay Triangulation" has the largest complexity: $\mathcal{O}\left(\mathrm{m}^{2}\right)$. This is due to the computation of the Delaunay triangulation in 3D [14]. Fortunately, the experimental complexity is almost linear in $m$ [10].

Step "Ray-Tracing" is $\mathcal{O}(m)$ since the ray length is bounded (using $\epsilon>0$, H4 and H5) and thanks to H1-3.

Step "2-Manifold Extraction" is $\mathcal{O}(m \log m)$. Indeed, there are $\mathcal{O}(m)$ tetrahedra (since $T$ has $\mathcal{O}(m)$ vertices $(\mathrm{H} 0)$ and $d$ is bounded $(\mathrm{H} 2)$ ) and we obtain the result thanks to Sec. 5.3 and H2.

Step "Topology Extension" is also $\mathcal{O}(m \log m)$. This is essentially due to the fact that the successive outside growings of this step are disjoint in the free-space. 


\begin{tabular}{|c|c|c|}
\hline 3D Delaunay Triangulation & DT & $\mathcal{O}\left(\mathrm{m}^{2}\right)$ \\
\hline Ray-Tracing & RT & $\mathcal{O}(m)$ \\
\hline Manifold Extraction & ME & $\mathcal{O}(m \log m)$ \\
\hline Topology Extension & TE & $\mathcal{O}(m \log m)$ \\
\hline Handle Removal (Sec. 4.2) & HR1 & N/A (see text) \\
\hline Handle Removal (Sec. 4.3) & HR2 & $\mathcal{O}(\mathrm{cm})$ \\
\hline Post Processing & PP & $\mathcal{O}(\mathrm{cm})$ \\
\hline
\end{tabular}

Table 1: Worst case time complexities and shortened names of all steps.

Step "Post-Processing" is $\mathcal{O}(\mathrm{cm})$, where $c$ is the number of camera locations $\mathbf{c}_{k}$. More precisely, Peak Removal and Surface Denoising are $\mathcal{O}(m)$, Sky Removal is $\mathcal{O}(\mathrm{cm})$. In practice, $c$ is quite smaller than the number $m$ of reconstructed vertices $(c<m / 85$ in our experiments).

Step "Handle Removal" in Sec. 4.2 has no "OO" additional complexity. Indeed, it adds to $T$ a number of Steiner vertices which is linear to $c$, and the complexities of all steps of the methods do not change since $c<m$.

Step "Handle Removal" in Sec. 4.3 is $\mathcal{O}\left(c m+w g_{0} \log g_{0}\right)$, where $w$ is the number of critical edges. Term $\mathrm{cm}$ is due to the detection of the critical edges; term $w g_{0} \log g_{0}$ is due to the force and repair operations (region growings whose sizes are bounded by $g_{0}$ ). In practice, the main term is $w g_{0} \log g_{0}$ where $g_{0}=10 d$ and $w$ is smaller than $m(w<m / 4.6$ in our experiments). We obtain complexity $\mathcal{O}(\mathrm{cm})$ using $w=\mathcal{O}(\mathrm{m})(\mathrm{H} 2)$ and $g_{0}=\mathcal{O}(1)$.

\section{Experiments}

Sec. 7.1 provides an overview of the results (both SfM and surface reconstruction) in the case of a still image sequence. Then we experiment and discuss the manifold constraint in Sec. 7.2, varying densities of reconstructed and Steiner points in Sec. 7.3 and 7.4, comparison with the Poisson surface reconstruction [21] in Sec. 7.5, handle removal in Sec. 7.6. Last, Sec. 7.7 gives quantitative experiments for a synthetic image sequence and Sec. 7.8 shows results for a $1.4 \mathrm{~km}$ long video sequence.

We use equiangular catadioptric cameras, which are hand/helmet-held. The view field is $360^{\circ}$ in the horizontal plane and about $50^{\circ}-60^{\circ}$ above and below. This is adequate for the $3 \mathrm{D}$ modeling of complete environments at 

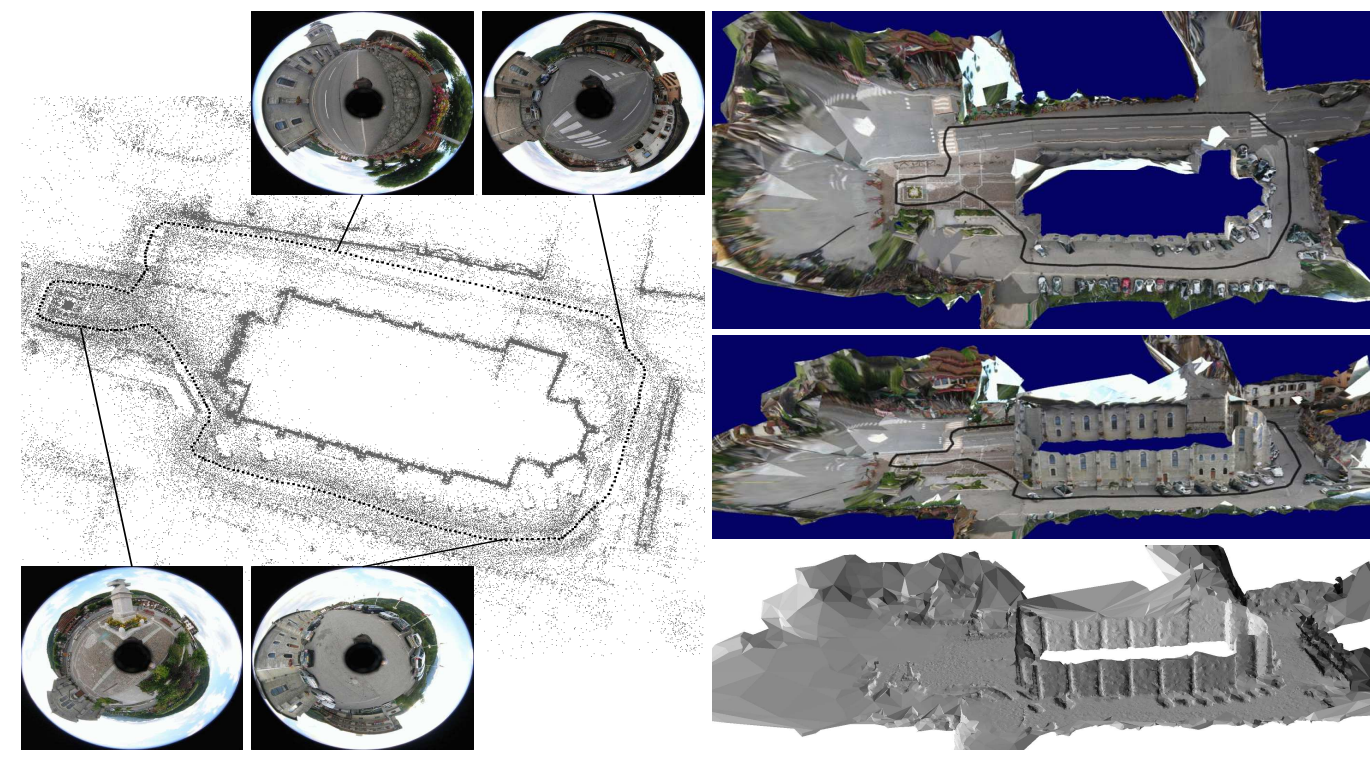

Figure 8: Overview of the results. Left: top view of the $136 \mathrm{k}$ points and 343 poses reconstructed by SfM and images of the sequence. Right: top and oblique views of the resulting surface (152k triangles), including triangle normals (gray) and trajectory (black).

low cost. The calculation times are given for an Intel Core 2 Duo E8500 at 3.16GHz. Suffix $k$ multiplies numbers by 1000 .

\subsection{Overview of the Results}

Except in Sec. 7.7 and 7.8, we use a still jpeg image sequence taken by the 0-360 mirror mounted with the Nikon Coolpix 8700 thanks to an additional adapter ring. We take 343 images during a complete walk around a church. Fig. 8 (left) shows top view of our SfM; it also estimates radial distortion parameters using a central model [24]. There are $136 \mathrm{k} 3 \mathrm{D}$ points and $872 \mathrm{k}$ Harris (inlier) points. The final RMS error is 0.74 pixels. A $2 \mathrm{D}$ point is considered as an outlier if the reprojection error is greater than 2 pixels.

Fig. 8 (right) shows the surface estimated using $\epsilon=10^{\circ}$ (Delaunay Step), without the Steiner vertices of the tight time complexity (Sec. 6), with the second Handle Removal method (HR2) and $\alpha=5^{\circ}$, and using post-processing thresholds: $w_{0}=\pi / 2$ steradians (Peak Removal), $\beta=45^{\circ}$ (Sky Removal). The parameter setting in this Section is the default setting of our paper. The VRML model is computed in 35s. It has $152 \mathrm{k}$ triangles, which are few for a scene like this. The lines "still" in Tab. 2 provides information for this 


\begin{tabular}{|c|c|c|c|c|c|c|c|c|}
\hline type & length & radius & $c$ & SfM & $|T|$ & $\frac{|F|}{|T|}$ & $\frac{\left|O_{\text {init }}\right|}{|F|}$ & $\frac{\left|O_{\text {end }}\right|}{|F|}$ \\
\hline still & $103 \mathrm{~m}$ & 574 pix. & 343 & $136 \mathrm{k}$ & $500 \mathrm{k}$ & 0.47 & 0.88 & 0.92 \\
\hline video & $1.4 \mathrm{~km}$ & 297 pix. & 2504 & $385 \mathrm{k}$ & $1335 \mathrm{k}$ & 0.51 & 0.87 & 0.92 \\
\hline \hline type & $|\delta O|$ & DT & RT & ME & TE & HR2 & PP & total \\
\hline still & $152 \mathrm{k}$ & $7.6 \mathrm{~s}$ & $2.9 \mathrm{~s}$ & $2.5 \mathrm{~s}$ & $2.9 \mathrm{~s}$ & $16.8 \mathrm{~s}$ & $2.5 \mathrm{~s}$ & $35 \mathrm{~s}$ \\
\hline video & $416 \mathrm{k}$ & $18.7 \mathrm{~s}$ & $6.9 \mathrm{~s}$ & $6.7 \mathrm{~s}$ & $5.9 \mathrm{~s}$ & $120 \mathrm{~s}$ & $7.7 \mathrm{~s}$ & $166 \mathrm{~s}$ \\
\hline
\end{tabular}

Table 2: Overview of our two sequences. Top: type, trajectory length, radius of large image circle, numbers of (key)frames and SfM points and tetrahedra, ratio of free-space tetrahedra, ratios of outside tetrahedra after Manifold Extraction and at the end. Bottom: number of triangles in the final VRML model, times for every step (shortening in Tab. 1).

sequence, including the computation times for every step, number of images, trajectory length, and performance of the outside growing in free-space (ratio between the numbers of free-space and outside tetrahedra). More details are given on several steps in the next Sections.

The joint video (supplementary content) has three parts: catadioptric images (input) and the corresponding panoramic images, walkthough in the 3D model from pedestrian's-eye views (the sky is not removed to obtain an immersive 3D model), full turn around the 3D model from bird's-eye view (the sky is removed). Both textures and triangle normals are shown in colors.

\subsection{2-Manifold Constraint}

Here are additional informations on the 2-manifold constraint in the experiment of Sec. 7.1. First, there are several methods to test that a surface is manifold (Sec. 2.3). We use the Single Tetrahedron Test for the 2-Manifold Extraction step (adding the tetrahedra one-by-one in $O$ ), and the GraphBased Test for the Topology Extension step (adding the tetrahedra severalat-once in $O$ ). In our implementation, the Polygon-Based Test is slower: if we use it, it multiplies the computation time of the 2-Manifold Extraction step by 1.4 and that of the Topology Extension step by 1.9. We also check that the different tests provide the same surface.

Now, we study the advantage of the 2-manifold constraint. We start from two closed surfaces: $\delta F$ (non-manifold) and $\delta O$ (manifold) generated by the 2-Manifold Extraction step. $24.6 \%$ of the $\delta F$ vertices are singular. Then the denoising step is used, and we obtain four surfaces: denoised manifold, nondenoised manifold, denoised non-manifold, non-denoised non-manifold. For 


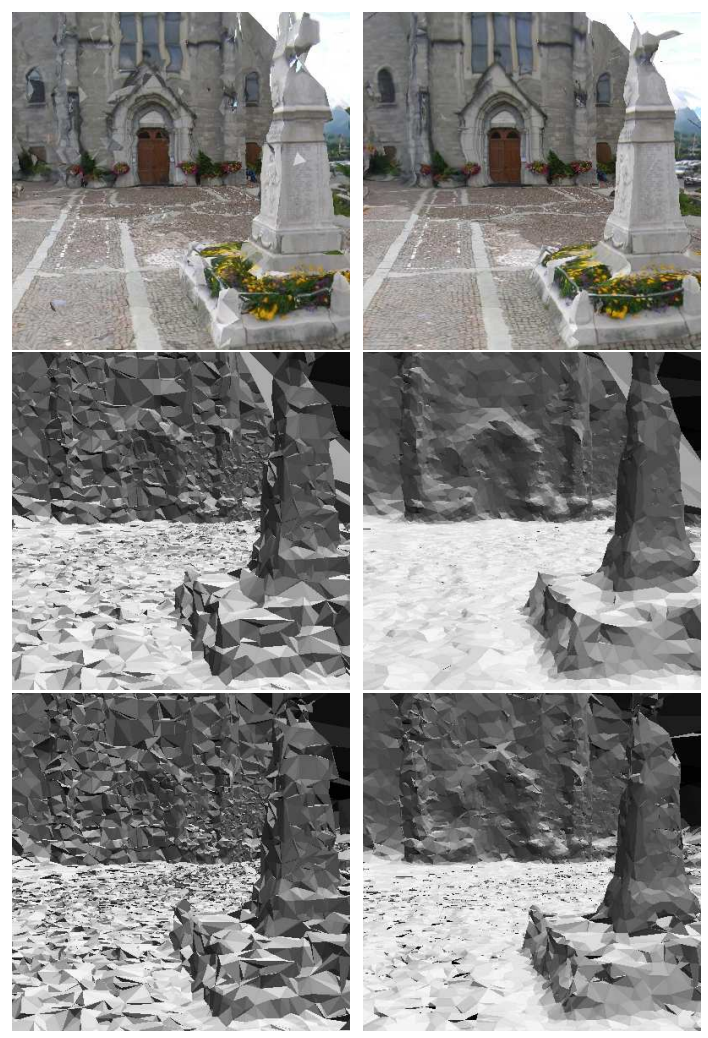

Figure 9: 2-manifold constraint and surface denoising. With (top and middle) and without (bottom) the manifold constraint. Without (left) and with (right) surface denoising.

the remainder of Sec. 7.2, the further steps of our method are not applied.

Fig. 9 shows that the manifold constraint helps surface denoising: the denoised manifold surface is smoother than the denoised non-manifold surface. The use of several denoising does not change this observation. Fig. 9 also shows that the texturing of the denoised 2-manifold is better than the texturing of the non-denoised 2-manifold.

\subsection{Density of Reconstructed Points}

Since our goal is the 2-manifold estimation from a small number of SfM points (and their visibility), it would be interesting to experiment on the same data as in Sec. 7.1, but with a yet smaller number of points. Thus we reduce the size of the images by several coefficients, and for each resulting sequence, we apply the whole process (both SfM and surface estimations) with the same parameter settings. This reduces the number of reconstructed 


\begin{tabular}{|c|c|c|c|c|c|c|}
\hline$r c$ & SfM & $m$ & $|T| / v$ & $\mathrm{~d}$ & $|\delta O|$ & $\mathrm{t}$ \\
\hline 1 & $136 \mathrm{k}$ & $81 \mathrm{k}$ & 6.19 & 296 & $152 \mathrm{k}$ & 35 \\
\hline 1.5 & $65 \mathrm{k}$ & $40 \mathrm{k}$ & 6.21 & 620 & $76 \mathrm{k}$ & 20 \\
\hline 2 & $37 \mathrm{k}$ & $23 \mathrm{k}$ & 6.22 & 386 & $44 \mathrm{k}$ & 12 \\
\hline 2.5 & $23 \mathrm{k}$ & $14 \mathrm{k}$ & 6.23 & 501 & $28 \mathrm{k}$ & 9.8 \\
\hline
\end{tabular}

Table 3: Numbers for calculations on reduced sequences: reduction coefficient, number of reconstructed points, number of Delaunay vertices, ratio between the numbers of tetrahedra and vertices, vertex degree, number of triangles, surface computation time in seconds.

points as if we use a different camera to reconstruct the same scene. Image reduction also increases the image noise impact on the 3D result. We think that this experiment is more interesting than the surface calculations from random selections of the SfM points in Sec. 7.1.

Tab. 3 provides numbers for reduction coefficients $r c \in\{1,1.5,2,2.5\}$. The number of reconstructed points is roughly linear to $1 /(r c)^{2}$, the ratio between the numbers of tetrahedra and vertices of the Delaunay is almost constant, and the calculation times are very small for large $r c$ (a few seconds). We also see that relations $|T|=\mathcal{O}\left(v^{2}\right)$ and $d=\mathcal{O}(v)$ are not tight enough to be used in the proofs of time complexities. Fig. 10 shows the surfaces for different $r c$ values. We see the progressive reduction of the level of details.

\subsection{Density of Steiner Vertices}

Here we study the effects of the Steiner vertices located at the corners of the Cartesian grid introduced in Sec. 6. The length of voxel edges of the grid is a multiple $s z$ of the camera step between two consecutive images (the length is $\left.s z \operatorname{mean}_{j}\left\|\mathbf{c}_{j+1}-\mathbf{c}_{j}\right\|\right)$.

Fig. 11 shows views for $s z \in\{2,5,10,20\}$. The smaller $s z$, the greater decrease of surface quality. The negative effect of the Steiner vertices is essentially in the areas where the density of reconstructed vertices is low (sky and parts of ground in our example). In the sky, there are too much tetrahedra for a fixed number of rays, then small matter tetrahedra appears if $s z$ is small. Thus $s z$ should not be too small.

Tab. 4 shows the experimental computation times for large enough $s z$ (the grid is unused if $s z=\infty$ ). We see that the grid does not really have complexity advantage in practice, and we conclude that the grid essentially has a theoretical interest: it guaranties the worst case complexities derived 


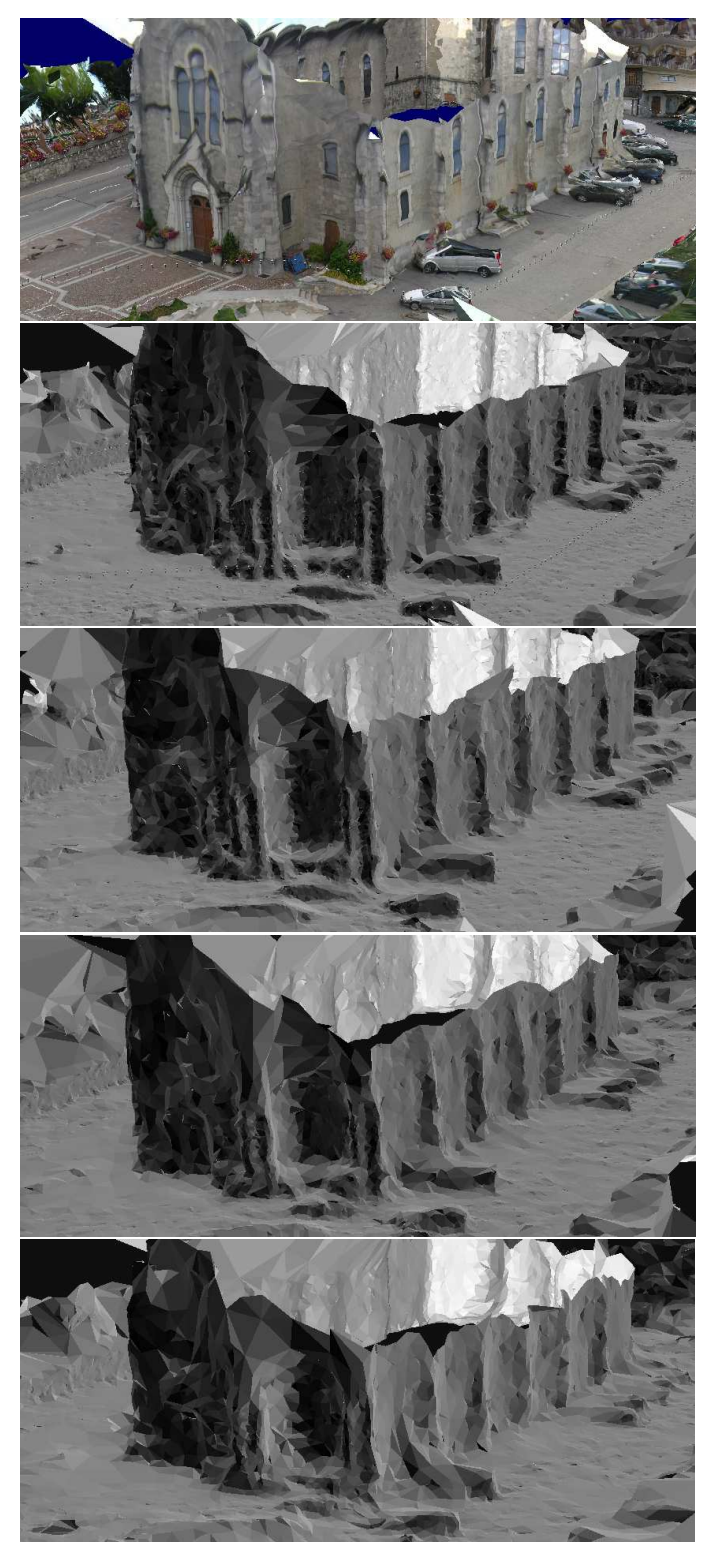

Figure 10: Surfaces for several densities of reconstructed points, defined by coefficient $r c$. From top to bottom: texture and normals for $r c=1,1,1.5,2,2.5$. 

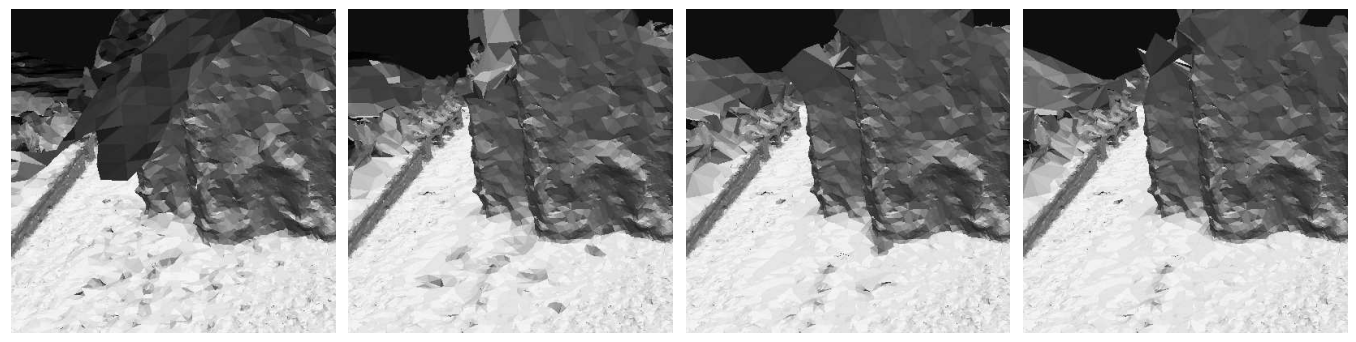

Figure 11: Effect of grids of Steiner vertices. From left to right: $s z=2,5,10,20$.

\begin{tabular}{|c|c|c|c|c|c|c|c|c|}
\hline$s z$ & $s / m$ & $|T| / v$ & $\mathrm{~d}$ & $\mathrm{DT}$ & $\mathrm{RT}$ & $\mathrm{ME}$ & $\mathrm{TE}$ & $\mathrm{HR} 2$ \\
\hline 10 & 0.079 & 6.11 & 386 & 9.0 & 2.7 & 2.4 & 2.2 & 15.1 \\
\hline 20 & 0.016 & 6.16 & 550 & 7.7 & 3.0 & 2.5 & 2.9 & 15.8 \\
\hline$\infty$ & 0 & 6.19 & 296 & 7.6 & 2.9 & 2.5 & 2.9 & 16.8 \\
\hline
\end{tabular}

Table 4: Numbers for several grids: edge length coefficient, ratio between the numbers of Steiner vertices and reconstructed vertices, ratio between numbers of tetrahedra and all vertices, vertex degree, time in seconds of steps DT...HR2 (shortening defined in Tab. 1).

in Sec. 6. The fact that the smallest $d$ in Tab. 4 is obtained without the grid might be surprising, but this is not in contradiction to the fact that every finite grid size $s z$ guaranties an upper bound for $d$ (Sec. 6.1). Note that $d$ can be smaller than $d(s z=\infty)$, e.g. $d(s z=5)=264$.

\subsection{Poisson Surface Reconstruction}

Our method is also compared to the Poisson Surface Reconstruction [21]. Remember that this method starts from a set of points and their oriented normals. We use parameter depth $=12$ to obtain a large enough resolution (the mesh size is moderated thanks to an octree-based sampling).

There are several surfaces depending on the method used to compute the oriented normal $\mathbf{n}_{i}$ of reconstructed point $\mathbf{p}_{i}$ :

- $S_{\text {ray }}: \mathbf{n}_{i}$ is the mean of directions of all $\mathbf{p}_{i}$ 's rays

- $S_{6 n n}: \mathbf{n}_{i}$ is the normal of the plane $\Pi$ minimizing the sum of the squared point-plane distances between $\Pi$ and the 6-nearest neighbors of $\mathbf{p}_{i}$

- $S_{\text {man }}: \mathbf{n}_{i}$ is a weighted sum of normals of the $\mathbf{p}_{i}$-incident triangles separating outside and inside tetrahedra. 

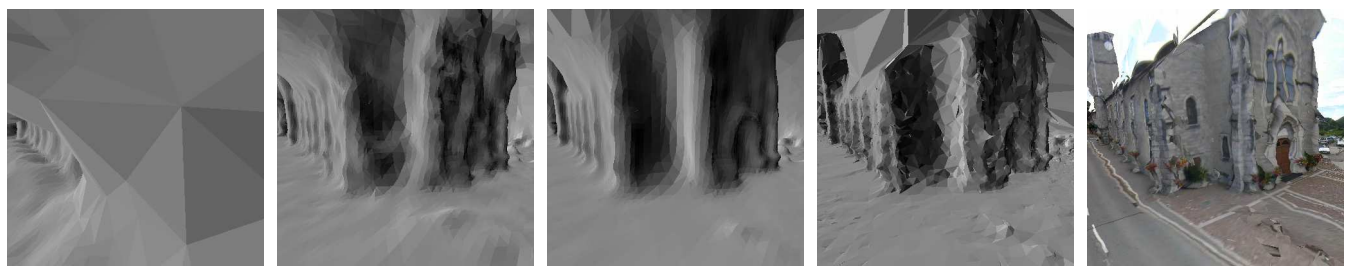

Figure 12: Comparison of Poisson's and ours surfaces. From left to right: $S_{\text {ray }}, S_{6 n n}$, $S_{\text {man }}, S_{\text {ours }}$ (normal) and $S_{\text {ours }}$ (texture) viewed from the same viewpoint.

And let $S_{\text {ours }}$ be the surface of our method.

In the four cases, the point filtering of Sec. 3.1 is applied and there is no Sky Removal step. Note that $S_{\text {man }}$ is obtained by a combination of two methods: ours and Poisson. Furthermore, the weight is the incident triangle angle at $\mathbf{p}_{i}$ [5], and $\mathbf{p}_{i}$ is not used by $S_{\text {man }}$ if its normal can not be computed due to the $\mathbf{p}_{i}$-tetrahedra labeling.

Fig. 12 shows views of the four surfaces. The better accuracy of normal computation, the better quality of the Poisson surface: $S_{m a n}$ is better than $S_{6 n n}$, which is better than $S_{\text {ray }}$. The quality of $S_{\text {ours }}$ is between those of $S_{6 n n}$ and $S_{m a n}$, although $S_{m a n}$ is oversmoothed compared to $S_{\text {ours }}$. Regarding the processing time, $S_{\text {ours }}$ has $154 \mathrm{k}$ triangles computed in $35 \mathrm{~s}$ and $S_{\text {man }}$ has $178 \mathrm{k}$ triangles computed in $35+93 \mathrm{~s}$, which is about 3.5 times of $S_{\text {ours }}$.

\subsection{Handle Removal}

Fig. 13 compares the two handle removal methods. HR1(ns) adds randomly a given number $n s$ of Steiner points in the ball neighborhood of every camera pose (Sec. 4.2). The radius coefficient is 10. HR2 detects, forces and repairs the spurious handles (Sec. 4.3). We compare HR2 to HR1(ns) with several values of $n s \in\{0,1,5,10\}$ (handles are not removed if $n s=0$ ). The right column shows the largest spurious handles of the surface if $n s=0$. These handles are reduced by HR1(1) and are removed by HR2 and HR1(ns), $n s \in\{5,10\}$. However, HR1(ns), $n s \in\{1,5,10\}$ are not able to remove spurious handles at other locations (two examples are shown in the two columns on the right). Furthermore, we notice that ns should not be too large for the same reasons as $s z$ should not be too small in Sec. 7.4. HR2 gives the best results and removes the large handles at the locations viewed by Fig. 13 (and others). Note that the sky is not removed in these experiments since we would like to examine the Handle Removal step only. Indeed, Sky Removal 

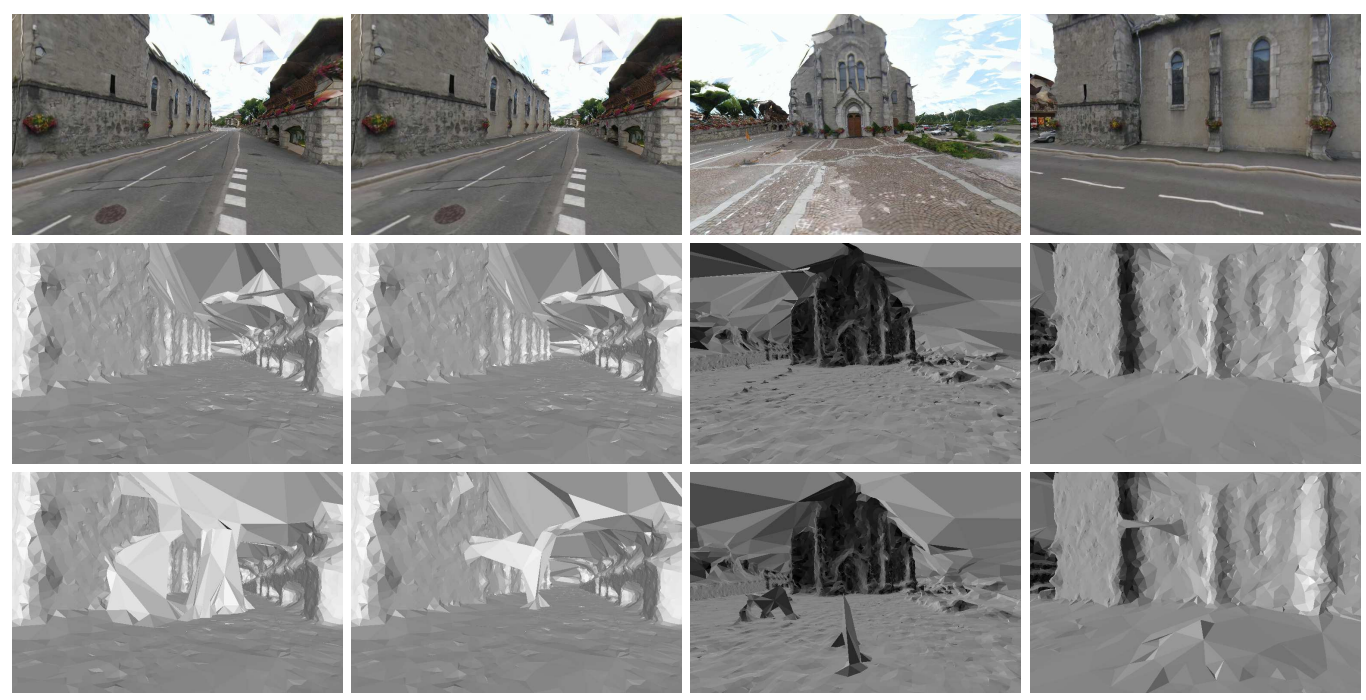

Figure 13: Comparison of two handle removal methods: HR1 (bottom) and HR2 (top and middle). Each column shows the results for given number of Steiner points per camera pose for HR1 (from left to right: $0,1,5,10$ ).

also removes spurious handles in a special case: if they are above the camera poses (they are intersected by the open rectangles of Sec. 3.5.3).

Remember that the main parameter of HR2 is the angle $\alpha$, which selects the minimal size of critical edges where the computations are done. We found that the $\alpha$ default value ( $5^{\circ}$ ) is a good compromise: (1) HR2 misses visually noticeable handles if $\alpha$ is larger than 5 and (2) the calculation times increases while the improvement is negligible if $\alpha$ is smaller than $5.1 .5 \%$ of the $581 \mathrm{k}$ Delaunay edges are critical edges using $\alpha=5$.

\subsection{Quantitative Evaluation on a Synthetic Scene}

The synthetic scene is manually generated from real images taken in a city. The trajectory is a $230 \mathrm{~m}$ long closed loop around a building including several shops. The images are generated by ray-tracing and taking into account the ray reflection on the catadioptric mirror. The large circle, which contains the scene projection in the image, has a 600 pixel radius. Fig. 14 (left) shows two images of the synthetic sequence. SfM [24] reconstructs 600 camera poses and a sparse cloud of $257 k$ 3D points from the sequence.

Now we explain how to estimate the reconstruction error. Let $Z$ be the similarity transformation which minimizes $E(Z)=\sum_{i=0}^{599}\left\|Z\left(\mathbf{c}_{i}\right)-\mathbf{c}_{i}^{g}\right\|^{2}$, where 

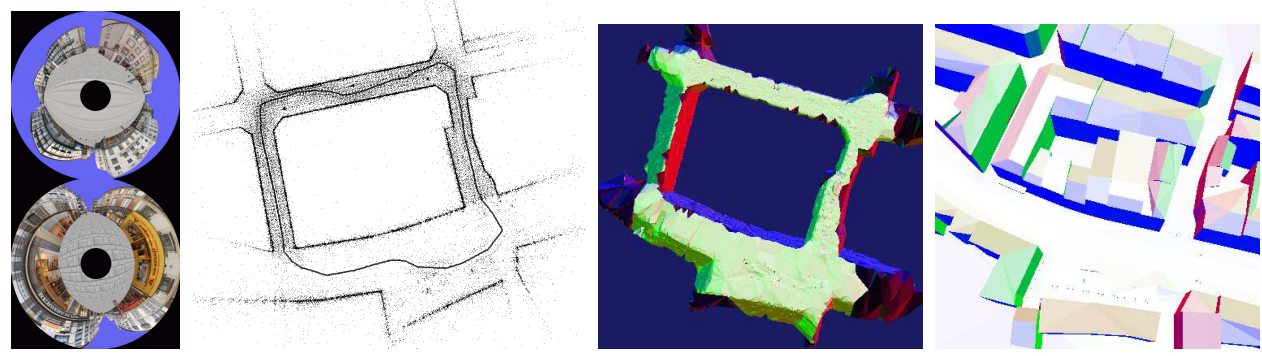

Figure 14: Two omnidirectional images, sparse SfM point cloud and camera trajectory, top view of the estimated and ground truth surfaces (the colors encode the triangle normal).

$\mathbf{c}_{i}$ and $\mathbf{c}_{i}^{g}$ are the estimated locations and the ground truth locations of the camera, respectively $\left(\mathbf{c}_{i}\right.$ is the camera center and $\mathbf{c}_{i}^{g}$ is the mirror apex). We use $Z$ to map the estimated geometry (poses and point cloud) in the ground truth coordinate system. Our error is based on ray tracing. Let $\mathbf{q}$ be a pixel. Let $\mathbf{p}_{e}$ be the intersection of the estimated surface and the back-projected ray of $\mathbf{q}$ by the estimated camera pose. Let $\mathbf{p}_{g}$ be the intersection of the ground truth surface and the back-projected ray of $\mathbf{q}$ by the ground truth camera pose. In both cases, if there are several intersections, we take the intersection which is the closest to the camera pose. Then we use error $e(\mathbf{q})=\left\|\mathbf{p}_{e}-\mathbf{p}_{g}\right\|$. If $\mathbf{p}_{g}$ does not exist or $e(\mathbf{q})>\mu_{0}$ (where $\mu_{0}=2 \mathrm{~m}$ ), we assume that the point matching $\left(\mathbf{p}_{e}, \mathbf{p}_{g}\right)$ is outlier (e.g. for the pixels of the sky).

The pose-registration provides $\sqrt{E(Z) / 600}=5.1 \mathrm{~cm}$. Then we uniformly sample pixels in the sequence and examine the distribution of $e(\mathbf{q})$ for 6000000 pixels. $75.7 \%$ of sampled points are inliers, the error median of inliers is $8 \mathrm{~cm}$, the $90 \%$ quantile of inliers is $55 \mathrm{~cm}$. Lastly, the same experiment (both SfM and surface estimations) is re-done for the same images down-sampled by 2 . We found $\sqrt{E(Z) / 600}=56 \mathrm{~cm}$, which implies that the SfM drift is larger than in the previous case. $73.9 \%$ of sampled points are inliers, the error median of inliers is $64.3 \mathrm{~cm}$, the $90 \%$ quantile of inliers is $103 \mathrm{~cm}$. Fig. 14 shows the sparse point cloud by SfM and the surface.

\subsection{Video as Input}

Here the catadioptric camera is the 0-360 mirror mounted with the Canon Legria HFS10. We take an AVCHD video biking in a city. The trajectory length is about $1.4 \mathrm{~km}$ long and the camera is mounted on a helmet.

Fig. 15 (top) shows a top view of the SfM result. First, a SfM method similar to [30] in the central case is applied. 2504 keyframes are selected 


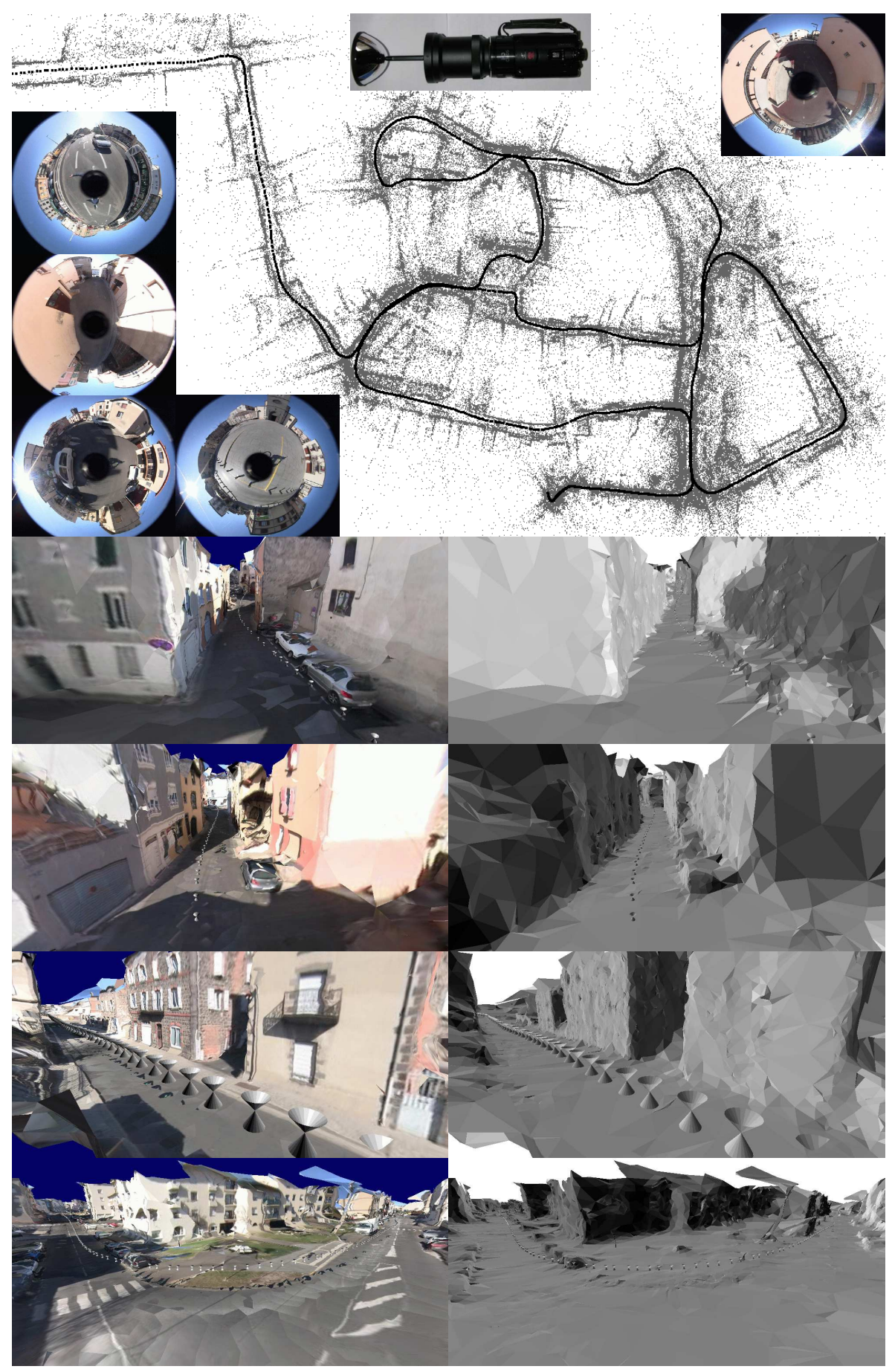

Figure 15: Top: top view of the SfM result, five images of the video sequence, and the catadioptric camera. Bottom: views of the VRML model (textures and triangle normals). 
from 24700 frames such that about 600 Harris points are matched in three consecutive keyframes. Second, we use the loop closure method in [25] (the SfM drift is not corrected at both sequence ends). We obtain 385k 3D points reconstructed from 2230k Harris (inlier) points in 2504 keyframes.

Fig. 15 (bottom) shows views of the VRML model with the parameters in Sec. 7.1. The 416k triangles of the surface are estimated in 166s. The lines "video" in Tab. 2 provides information for this sequence, including the computation times for every step. The most costly step is Handle Removal; $3 \%$ of the $1548 \mathrm{k}$ Delaunay edges are critical edges.

The mean number of points reconstructed per meter is 275 (ratio between SfM and length in Tab. 2). Thus the scene model is very simplified and compact. The thin details such as electric posts, threes with low density foliage, window concavities in facades, are not modeled. Note that this number (275) is quite smaller than that of the "still" sequence (1320). Both image resolution and number of points contribute to the surface quality.

\section{Conclusion}

In this paper, a 2-manifold surface is reconstructed from the Structurefrom-Motion output: a sparse cloud of reconstructed Harris points and their visibilities in the images. The potential adjacencies between the points are encoded in a 3D Delaunay triangulation, then the point visibilities are used to label the tetrahedra, last a 2-manifold is extracted thanks to a greedy method and the use of Steiner vertices. The time complexity is also studied. Furthermore, the experiments show the interest of the manifold constraint (neglected in the previous work using the same sparse SfM data), results using several densities of reconstructed/Steiner vertices, comparisons to the Poisson surface reconstruction, comparisons between variants of our method, quantitative evaluation, both still and video (catadioptric) image sequences.

The current system is able to reconstruct the main components of environments (ground, building, dense vegetation ...), but the main limitation is due to the lack of points to reconstruct thin scene details. Future work can improve the surface quality while staying in our sparse feature scheme and maintaining a low computational cost: reconstruct and integrate the image contours in the 3D Delaunay triangulation, improve the interest point matching (especially in the video case), design a dedicated surface denoising method for sparse SfM point clouds, investigate other cost functions $r$. Otherwise, the accuracy could be improved by a dense stereo refinement. 

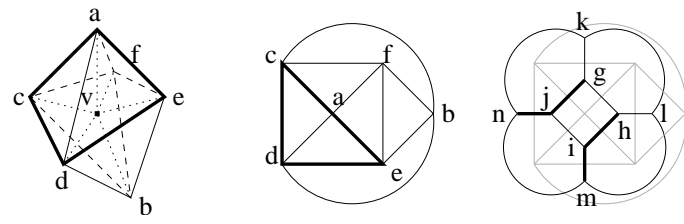

Figure A.16: Duality of planar graphes. Left: the height v-incident tetrahedra whose union is an octahedron, and border (bold edges) between inside and outside tetrahedra. Middle: planar graph $G$ and bold edges $F$. Right: planar graph $G^{*}$ and bold edges $F^{*}$. The dual of $G$ (octahedron) is $G^{*}$ (cube). The dual of $F=\{a c, c d, d e, e a\}$ is $F^{*}=\{j g, j n, i m, i h\}$.

\section{Appendix A. Sketch Proof of the Graph-Based Test}

Here we use theory on planar graphs and duality in [33] to show that the Graph- and Edge-based tests in Sec. 2.3 are equivalent.

First, we introduce notations $G$ and $G^{*}$. Let $\mathbf{v}$ be a vertex on surface $\delta O$. Let $V^{*}$ be the list of $\mathbf{v}$-incident tetrahedra of the Delaunay $T$. Let $E^{*}$ be the list of triangles between adjacent tetrahedra in $V^{*}$. Graph $G^{*}=\left(V^{*}, E^{*}\right)$ has vertices $V^{*}$ and edges $E^{*}$, and $G^{*}=g_{\mathbf{v}}$. Let $V$ be the list of vertices of the tetrahedra of $V^{*}$, except $\mathbf{v}$. Let $E$ be the list of edges of the tetrahedra of $V^{*}$ without endvertex $\mathbf{v}$. Graph $G=(V, E)$ has vertices $V$ and edges $E$.

Fig. A.16 shows an example: there are height $\mathbf{v}$-incident tetrahedra whose union is an octahedron centered at $\mathbf{v}, G$ has the vertices and edges of the octahedron, $G^{*}$ has the vertices and edges of a cube. Note that $G^{*}$ is also the adjacency graph of the $\mathbf{v}$-opposite triangles in the $\mathbf{v}$-incident tetrahedra.

Second we detail the duality between $G$ and $G^{*}$. Graph $G$ is planar since it lies on the border of a $\mathbf{v}$-star-shaped volume which is homeomorphic to a 2-sphere. Remember that a planar graph is embedded in $\mathbb{R}^{2}$, it cuts $\mathbb{R}^{2}$ into regions called faces such that every edge has two faces (one on the left and one on the right). The dual graph of $G$ is defined as follows: its vertices are the faces of $G$, its edges connect the left and right faces of an edge in $G$. Now we see that $G^{*}$ is the dual graph of $G$.

Third we explicit the duality between edges due to the inside-outside labeling. We have a nontrivial bipartition of $V^{*}: V_{I}^{*}$ (inside tetrahedra) and $V_{O}^{*}$ (outside tetrahedra). This implicitly defines a bipartition of inside and outside (v-opposite) triangles. Let $F^{*}$ be the edges of graph $G^{*}$ having an endvertex in $V_{I}^{*}$ and an endvertex in $V_{O}^{*}$. Let $F$ be the edges of graph $G$ having an inside triangle and an outside triangle as its two faces. The edges $F^{*}$ of graph $G^{*}$ are the duals of edges $F$ of graph $G$ (Fig. A.16). 
Last we show that Graph-based and Edge-based tests give the same result. According to the Graph-based test, $\mathbf{v}$ is regular iff (if and only if) $V_{I}^{*}$ and $V_{O}^{*}$ are connected in the graph $\left(V^{*}, E^{*} \backslash F^{*}\right)$, i.e. iff $F^{*}$ is a minimal edge-cut of $G^{*}$. According to the Edge-based test, $\mathbf{v}$ is regular iff $F$ is a cycle in graph $G$. Now we use proposition 7 in [33]: "Let $G=(V, E)$ be a connected plane multigraph. A set $F$ included in $E$ is a cycle in $G$ if and only if $F^{*}$ is a minimal edge-cut in $G^{*}$ ". We obtain the result. Note that a multigraph is a graph allowing loop edges and parallel edges, that our graphes have not.

\section{Appendix B. Single Tetrahedron Test}

Assume that $\delta O$ is a 2-manifold. In Sec. 2.3.4, we would like to add one tetrahedron $\Delta$ in $O$ such that $\delta O$ remains a 2-manifold using the structures in Sec. 5.1. Let $f$ be the number of $\Delta$ facets (i.e. triangles) which are included in $\delta O$. If $f=0, \Delta$ will be added to $O$ if and only if the $\Delta$ vertices do not have incident tetrahedron in $O$. If $f=1, \Delta$ will be added to $O$ if and only if the $\Delta$ vertex, which is not in the $\Delta$ facet included in $\delta O$, does not have incident tetrahedron in $O$. If $f=2, \Delta$ will be added to $O$ if and only if the $\Delta$ edge, which is not an edge of the two $\Delta$ facets included in $\delta O$, does not have incident tetrahedron in $O$. If $f \in\{3,4\}, \Delta$ will be added to $O$.

The complexity of this step is needed in Sec. 5.2. Cases $f \in\{3,4\}$ are $\mathcal{O}(1)$. Since the computation of the list $L_{\mathbf{v}}$ of tetrahedra incident to a (finite) vertex $\mathbf{v}$ is a traversal of graph $g_{\mathbf{v}}$, it is $\mathcal{O}(d)$. Then cases $f \in\{0,1\}$ are $\mathcal{O}(d)$. Case $f=2$ requires the computation of the list $L_{e}$ of tetrahedra which include an edge $e$. Let $\mathbf{v}_{1}$ and $\mathbf{v}_{2}$ be the endvertices of $e$. Since $\Delta^{\prime} \in L_{e}$ if and only if $\Delta^{\prime} \in L_{\mathbf{v}_{1}}$ and $\Delta^{\prime}$ has vertex $\mathbf{v}_{2}$, case $f=2$ is also $\mathcal{O}(d)$.

In practice, we greatly accelerate these computations and others by precalculating for every vertex the list (stored as a table) of its incident tetrahedra. This is done in $\mathcal{O}(|T|)$ from the data structure in Sec. 5.1.

\section{Appendix C. Peak Removal can Remove Spurious Concavity}

Fig. C.17 explains how Peak Removal (Sec. 3.5.1) can remove a spurious concavity due to bad point $\mathbf{p}$ (Sec. 3.6). Here $\mathbf{p}$ carves a peak due to its rays, the peak apex is $\mathbf{p}$ and the acute side of the peak is the spurious concavity. The larger density of the good points, the smaller the angle of the peak, the better is the success rate of the spurious concavity removal. 


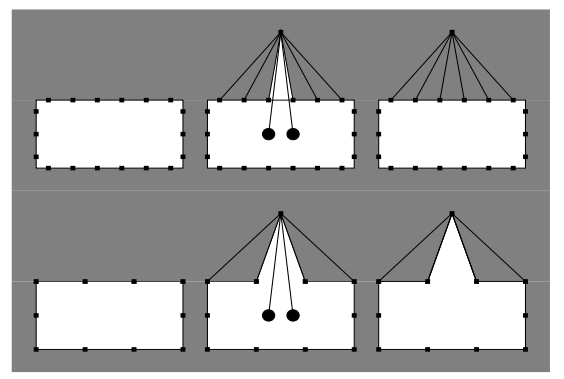

Figure C.17: Peak Removal can remove spurious concavity (2D case). Outside is white, inside is gray. Points and camera locations are small black squares and large disks, respectively. The density of good points on the surface is high (top) or low (bottom). Left: no bad point. Middle: one bad point adds a spurious concavity due to its two rays. Right: Peak Removal removes the concavity (which is a peak) if it has a small angle.

\section{Appendix D. Proof of the Bounded Density of the Tetrahedra}

Since $T$ is Delaunay, every tetrahedron has a circumscribing sphere such that the interior does not contain any Delaunay vertex [4]. These vertices include the Steiner vertices of the grid introduced in Sec. 6.1. Then Appendix D.1 shows the intuitive result that the tetrahedron diameter is less than (or equal to) the diagonal length of a grid voxel. Last we finish the proof thanks to Appendix D.2.

\section{Appendix D.1. Bounded Diameter of Tetrahedron}

To make the proof easier, the grid is increased by an additional layer of Steiner vertices as shown in Fig D.18. Let $A=\{-1,0,1,2\}$. Without loss of generality, the grid contains vertices $A^{3}=A \times A \times A$, point $\mathbf{p}$ is in the cube $[0,1]^{3}$, the length of the cube diagonal is $\sqrt{3}$. Let $\mathbf{c}$ be the center of a circumscribing sphere $\mathcal{S}$ of a tetrahedron which has vertex p. Here we show that $\|\mathbf{c}-\mathbf{p}\| \leq \sqrt{3} / 2$ (then the tetrahedron diameter is less than $\sqrt{3}$ ).

Assume (reductio ad absurdum) that $\mathbf{c} \notin[-1,2]^{3}$. We show that

$$
\min _{\mathbf{s} \in A^{3}}\|\mathbf{c}-\mathbf{s}\|<\|\mathbf{c}-\mathbf{p}\| .
$$

Using coordinates of vectors $\mathbf{c}, \mathbf{s}$ and $\mathbf{p}$, Eq. D.1 means that

$$
\min _{s_{x} \in A}\left(c_{x}-s_{x}\right)^{2}+\min _{s_{y} \in A}\left(c_{y}-s_{y}\right)^{2}+\min _{s_{z} \in A}\left(c_{z}-s_{z}\right)^{2}
$$

is less than

$$
\left(c_{x}-p_{x}\right)^{2}+\left(c_{y}-p_{y}\right)^{2}+\left(c_{z}-p_{z}\right)^{2} .
$$




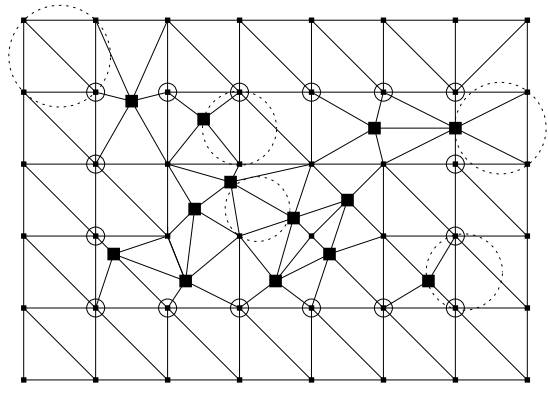

Figure D.18: Delaunay mesh $T$ (in the 2D case) for tight complexity analysis. Steiner and reconstructed points are small and large black squares, respectively. The dotted circles are circumcircles of triangles. The surrounded points by small circle are the border of the bounding box. This box is increased by an additional layer of Steiner vertices.

Using $u \in\{x, y, z\}$, we introduce

$$
f_{u}=\left(c_{u}-p_{u}\right)^{2}-\min _{s_{u} \in A}\left(c_{u}-s_{u}\right)^{2} .
$$

It can be shown that

$$
\begin{aligned}
p_{u} \in[0,1] \text { and } c_{u} \in[-1,2] & \Rightarrow f_{u} \geq-\min _{s_{u} \in A}\left(c_{u}-s_{u}\right)^{2} \geq-1 / 4 \\
p_{u} \in[0,1] \text { and } c_{u} \leq-1 & \Rightarrow f_{u}=\left(c_{u}-p_{u}\right)^{2}-\left(c_{u}+1\right)^{2} \geq 1 \\
p_{u} \in[0,1] \text { and } 2 \leq c_{u} & \Rightarrow f_{u}=\left(c_{u}-p_{u}\right)^{2}-\left(c_{u}-2\right)^{2} \geq 1
\end{aligned}
$$

Since $\mathbf{c} \notin[-1,2]^{3}$, there is $u \in\{x, y, z\}$ such that $c_{u} \notin[-1,2]$. Thus $f_{x}+$ $f_{y}+f_{z} \geq 1-1 / 4-1 / 4>0$ and Eq. D. 1 is met.

However, $\|\mathbf{c}-\mathbf{p}\|$ is the $\mathcal{S}$ radius and Eq. D.1 means that the interior of $\mathcal{S}$ contains a vertex s of the grid. This is not possible in a 3D Delaunay triangulation [4]. Thus assumption $\mathbf{c} \notin[-1,2]^{3}$ is wrong.

Since $\mathbf{c} \in[-1,2]^{3}, \mathbf{c}$ is in a cube of $A^{3}$ grid. So there is a vertex $\mathbf{s} \in A^{3}$ such that $\|\mathbf{s}-\mathbf{c}\|^{2} \leq(1 / 2)^{2}+(1 / 2)^{2}+(1 / 2)^{2}$, i.e. $\|\mathbf{s}-\mathbf{c}\| \leq \sqrt{3} / 2$. Since the interior of $\mathcal{S}$ does not contains $\mathbf{s}$, we see that $\|\mathbf{c}-\mathbf{p}\| \leq\|\mathbf{s}-\mathbf{c}\| \leq \sqrt{3} / 2$.

\section{Appendix D.2. From Bounded Diameter to Bounded Density of Tetrahedra}

Let $l$ be the diagonal length of a grid voxel. Let $p^{\prime}>0$ and $L(\mathbf{z})$ be the list of tetrahedra which intersect the $p^{\prime}$-ball centered at point $\mathbf{z}$. Thanks to the triangular inequality, the tetrahedra of $L(\mathbf{z})$ are in the $p^{\prime}+l$-ball centered at $\mathbf{z}$. In $\mathbb{R}^{3}$, we can cover the $p^{\prime}+l$-ball with a finite number $b$ of $p$-balls ( $b$ does not depend on $\mathbf{z}$ ). According to Sec. 6.1, each $p$-ball contains at 
most $q$ vertices. Thus the $p^{\prime}+l$-ball contains at most $b q$ vertices. Since a tetrahedron is a 4-tuple of vertices, we see that the number of tetrahedra in $L(\mathbf{z})$ is bounded. We conclude that the tetrahedron density is bounded.

\section{Appendix E. Proofs of Complexities}

Here are the proofs for results in Sec. 6.3 using assumptions in Sec. 6.2.

\section{Appendix E.1. "Ray-Tracing" Complexity}

Firstly, we estimate the complexity of tracing one ray (line segment) $\mathbf{c}_{j} \mathbf{p}_{i}$. The ray is covered by a number of $p^{\prime}$-balls which is linear to the euclidean distance $\left\|\mathbf{c}_{j}-\mathbf{p}_{i}\right\|$. Since every $p^{\prime}$-ball intersects at most $q^{\prime}$ tetrahedra (H1), $\mathbf{c}_{j} \mathbf{p}_{i}$ intersects a number of tetrahedra which is $\mathcal{O}\left(\left\|\mathbf{c}_{j}-\mathbf{p}_{i}\right\|\right)$. Furthermore, $\mathbf{c}_{j} \mathbf{p}_{i}$-tracing is a walk (Sec. 3.2) in graph $T$ restricted to these tetrahedra and started from $\mathbf{p}_{i}$, which is a $T$ vertex. Now we see that $\mathbf{c}_{j} \mathbf{p}_{i}$-tracing has time complexity $\mathcal{O}\left(\left\|\mathbf{c}_{j}-\mathbf{p}_{i}\right\|\right)$ thanks to $\mathrm{H} 3$.

Secondly, we show that ray length $\left\|\mathbf{c}_{j}-\mathbf{p}_{i}\right\|$ is bounded. Let $\mathbf{h}$ be the orthogonal projection of $\mathbf{c}_{j}$ into the line crossing $\mathbf{c}_{k}$ and $\mathbf{p}_{i}$. This implies $\left\|\mathbf{h}-\mathbf{c}_{j}\right\| \leq\left\|\mathbf{c}_{k}-\mathbf{c}_{j}\right\|$ and $\sin \left(\widehat{\mathbf{c}_{j} \mathbf{p}_{i} \mathbf{c}_{k}}\right)=\left\|\mathbf{h}-\mathbf{c}_{j}\right\| /\left\|\mathbf{p}_{i}-\mathbf{c}_{j}\right\|$. Furthermore, reconstructed point $\mathbf{p}_{i}$ is added to $T$ if it meets the ray-angle criterion $\pi-\epsilon \geq$ $\widehat{\mathbf{c}_{j} \mathbf{p}_{i} \mathbf{c}_{k}} \geq \epsilon>0$ (Sec. 3.1). We obtain

$$
\left\|\mathbf{p}_{i}-\mathbf{c}_{j}\right\|=\frac{\left\|\mathbf{h}-\mathbf{c}_{j}\right\|}{\sin \widehat{\mathbf{c}_{j} \mathbf{p}_{i} \mathbf{c}_{k}}} \leq \frac{\left\|\mathbf{c}_{k}-\mathbf{c}_{j}\right\|}{\sin \epsilon}
$$

Now, H4 and H5 imply that $\left\|\mathbf{c}_{j}-\mathbf{c}_{k}\right\|$ is bounded if $j \in V_{i}$ and $k \in V_{i}$. Thus, $\left\|\mathbf{p}_{i}-\mathbf{c}_{j}\right\|$ is also bounded and $\mathbf{c}_{j} \mathbf{p}_{i}$-tracing is $\mathcal{O}(1)$.

Lastly, the time complexity is $\mathcal{O}(m)$ since there are $m$ reconstructed vertices $(\mathrm{H} 0)$, every vertex has $\mathcal{O}(1)$ rays $(\mathrm{H} 4)$, and tracing one ray is $\mathcal{O}(1)$.

\section{Appendix E.2. "Topology Extension" Complexity}

Remember that Topology Extension alternates two steps:

1. switching from inside to outside tetrahedra around a surface vertex

2. region growing in $F$ starting from the neighborhood of these tetrahedra.

Step 2 only occurs if step 1 is successful. If it is not successful, step 1 includes the reverse switching (from outside to inside). This process is tried a finite number of times for every surface vertex. 
There are $\mathcal{O}(m)$ surface vertices, and $\mathrm{H} 2-\mathrm{H} 3$ imply that step 1 is $\mathcal{O}(1)$ for one vertex. So the complexity of all steps 1 is $\mathcal{O}(m)$. The i-th growing from the i-th vertex (step 2) has complexity $\mathcal{O}\left(\left(g_{i}+q_{i}\right)\left(d+\log \left(g_{i}+q_{i}\right)\right)\right)$ where $g_{i}$ is the number of grown tetrahedra and $q_{i}$ the number of tetrahedra in $Q_{0}$. We have $\sum_{i} g_{i}=\mathcal{O}(m)$ since the total number of tetrahedra is $\mathcal{O}(m)$. We have $\sum_{i} q_{i}=\sum_{i} \mathcal{O}(1)=\mathcal{O}(m)$ since $q_{i}=\mathcal{O}(1)$ thanks to H2. Thus,

$$
\begin{aligned}
\sum_{i \in I}\left(g_{i}+q_{i}\right)\left(d+\log \left(g_{i}+q_{i}\right)\right) & \leq \sum_{i \in I}\left(g_{i}+q_{i}\right)\left(d+\log \left(\sum_{i^{\prime} \in I} g_{i^{\prime}}+\sum_{i^{\prime} \in I} q_{i^{\prime}}\right)\right) \\
& =\mathcal{O}(m(d+\log m) .
\end{aligned}
$$

Thanks to H2, the complexity of all steps 2 is $\mathcal{O}(m \log m)$. We see that Topology Extension is $\mathcal{O}(m \log m)$.

\section{Appendix E.3. "Post-Processing" Complexity}

Trying to remove one peak $\mathbf{p}_{i}$ of the surface $S$ is $\mathcal{O}(1)$ : the access to the data (tetrahedra and vertices) to do this is $\mathcal{O}(1)$ thanks to $\mathrm{H} 2$ and $\mathrm{H} 3$, the manifold test at a vertex is $\mathcal{O}(1)$ thanks to $\mathrm{H} 2$. Since $S$ has $\mathcal{O}(m)$ vertices and every vertex is tried a finite number of times, Peak Removal is $\mathcal{O}(m)$.

Remember that $\mathbf{p}^{\prime}$ is calculated from $\mathbf{p}$ and list $\mathcal{N}(\mathbf{p})$ of vertices which are connected to $\mathbf{p}$ by an edge of $S$. Thanks to $\mathrm{H} 2$ and $\mathrm{H} 3$, the calculation of $\mathcal{N}(\mathbf{p})$ is $\mathcal{O}(1)$. Since $S$ has $\mathcal{O}(m)$ vertices, Surface Denoising is $\mathcal{O}(m)$.

Now we study Sky Removal. The rectangle-triangle intersections are $\mathcal{O}(\mathrm{cm})$ since $S$ has $\mathcal{O}(\mathrm{m})$ triangles (thanks to $\mathrm{H} 2$ ) and there are $c$ open rectangles. The border growing is bounded by the number of $S$ triangles. Thus Sky Removal is $\mathcal{O}(\mathrm{cm})$.

\section{Appendix E.4. "Spurious Handle Removal" Complexity}

The first method (Sec. 4.2) adds to $T$ a number of Steiner vertices which is linear to $c$. In practice, there are no more than 10 Steiner vertices per camera location and they act as reconstructed vertices with empty visibility lists. Since $c<m$, the complexities of all steps of the methods do not change.

Now we study the second method (Sec. 4.3). Thanks to H2, $T$ has $\mathcal{O}(m)$ edges and the complexity of step "Detect" for every edge is $\mathcal{O}(d+c)=\mathcal{O}(c)$. Then "Detect" is $\mathcal{O}(\mathrm{cm})$.

In the "Force" step, the vertices of the split edges are finite. Then the complexity of one split edge is $\mathcal{O}(1)$ thanks to $\mathrm{H} 2$ and H3. Furthermore, every

critical edge generates 3 vertices $\mathbf{v}_{i}$, and the time and space complexities of 
$L_{i}$ is $\mathcal{O}(1)$ for every $\mathbf{v}_{i}$ (thanks to $\mathrm{H} 2$ and H3). We see that the computation time of "Force" for one critical edge is $\mathcal{O}(1)$. Thus the Force complexity is $\mathcal{O}(w)$, where $w$ is the number of critical edges.

"Repair" is a best first region growing which increases $O$ using a heap $Q$ as the method in Sec. 3.3. Thus, it can be shown (as in Sec. 5.3) that the number of "while" iterations is $\mathcal{O}\left(g+q_{0}\right)$ where $g$ is the number of grown tetrahedra and $q_{0}$ the number of tetrahedra in the initialization of heap $Q$. Furthermore, the complexity of one "while" iteration is $\mathcal{O}\left(d+\log \left(g+q_{0}\right)\right)$ as in Sec. 5.3. In the Repair case, $q_{0}=\mathcal{O}(1)$ (since $\left.\left|L_{i}\right|=\mathcal{O}(1)\right)$ and $g \leq g_{0}$. We see that the complexity of one "Repair" is $\mathcal{O}\left(g_{0} \log g_{0}\right)$. There are $\mathcal{O}(1)$ uses of "Repair" for every critical edge since $\left|L_{i}\right|=\mathcal{O}(1)$. Thus the complexity of all "Repair" uses is $\mathcal{O}\left(w g_{0} \log g_{0}\right)$. We conclude that the second removal method is $\mathcal{O}\left(\mathrm{cm}+w g_{0} \log g_{0}\right)$.

\section{Acknowledgments}

Thanks to CNRS, Université Blaise Pascal (UBP) and Institut Pascal for funding Maxime Lhuillier and Shuda Yu. Thanks to the CRISTAL project for providing the ground truth VRML model used in the quantitative evaluation.

\section{References}

[1] CGAL. Computational Geometry Algorithm Library. www.cgal.org

[2] N. Amenta and M. Bern. Surface reconstruction by voronoi filtering. Discrete Computational Geometry, 22, pp. 481-504, 1999.

[3] M. Bern and F. Eppstein and J. Gilbert. Provably good mesh generation. Computer and System Sciences, 48(3), pp. 384-409, 1994.

[4] J. Boissonnat. Geometric structures for three-dimensional shape representation. ACM Transactions on Graphics, 3(4):266-286, 1984.

[5] M. Botsch, L. Kobbelt, M. Pauly, P. Alliez, and B. Levy. Polygon Mesh Processing. AK Peters, 2010.

[6] F. Cazals and J. Giesen. Delaunay triangulation based surface reconstruction: ideas and algorithms, 2004. INRIA technical report 5394. 
[7] B. Charmette, E. Royer and F. Chausse. Matching Planar Features for Robot Localization. In Advances in Visual Computing, 2009. Also in LNCS 5875.

[8] N. Cornelis, K. Cornelis and L. Van Gool. Fast Compact City Modeling for Navigation Pre-Visualization. In CVPR'06.

[9] P. Doubek and T. Svoboda. Reliable 3D-reconstruction from a few catadioptric images. In OMNIVIS'02.

[10] O. Faugeras, E. L. Bras-Mehlman, and J. Boissonnat. Representing stereo data with the delaunay triangulation. Artificial Intelligence, pp. 41-47, 1990. Also in INRIA technical report 788.

[11] O. Faugeras and R. Keriven. Complete Dense Stereovision using Level Set Methods. In CVPR'98.

[12] Y. Furukawa and J. Ponce. Accurate, Dense, and Robust Multi-View Stereopsis. In CVPR'O\%.

[13] A. Gueziec, G. Taubin, F. Lazarus and B. Horn. Cutting and Stitching: Converting Sets of Polygons to Manifold Surfaces. IEEE Transaction on Visualisation and Computer Graphics, 7(2), pp. 136-151, 2001.

[14] J. Goodman and J.O.'Rourke (editors). Handbook of Discrete and Computational Geometry. Second Edition, CRC Press, 2004.

[15] C. Harris and M. Stephens. A Combined Corner and Edge Detector. In Alvey Vision Conf., pp. 147-151, 1988.

[16] R. Hartley and A. Zisserman. Multiple View Geometry in Computer Vision. Cambridge University Press, 2003.

[17] C.H.Esteban and F. Schmitt. Silhouette and Stereo Fusion 3D Object Modeling. CVIU, 96(3), pp. 367-392, 2004.

[18] V. Hiep, R. Keriven, P. Labatut, and J. Pons. Towards high-resolution large-scale multi-view stereo. In CVPR'09.

[19] A. Hilton. Scene modelling from sparse 3D data. Image and Vision Computing, 23, pp. 900-920, 2005. 
[20] M. Jancosek and T. Pajdla. Multi-View Reconstruction Preserving Weakly-Supported Surfaces. In CVPR'11.

[21] M. Kazhdan, M. Bolitho, and H. Hoppe. Poisson surface reconstruction. In Eurographics Symposium on Geometry Processing'06.

[22] P. Labatut, J. Pons, and R. Keriven. Efficient multi-view reconstruction of large-scale scenes using interest points, Delaunay triangulation and Graph-Cuts. In CVPR'O\%.

[23] M. Lhuillier and L. Quan, A Quasi-Dense Approach to Surface Reconstruction from Uncalibrated Images. TPAMI, 27(3), pp. 418-433, 2005.

[24] M. Lhuillier. Automatic Scene Structure and Camera Motion using a Catadioptric System. CVIU, 109(2), pp. 186-203, 2008.

[25] M. Lhuillier. Environment Modeling from Images taken by a Low Cost Camera. International Archives of Photogrammetry, Remote Sensing and Spatial Information Sciences, vol. XXXVIII part 3A, 2010 (also in proceedings of PCV'10).

[26] W. Lorensen and H. Cline. A high resolution 3D surface construction algorithm. In SIGGRAPH'8\%.

[27] D. Lovi, N. Birkbeck, D. Cobzas, and M. Jagersand. Incremental freespace carving for real-time 3D reconstruction. In 3DPVT'10.

[28] M. Meyer, M. Desbrun, P. Schroder, and A. Barr. Discrete differentialgeometry operators for triangulated 2-manifolds. In Visualization and Mathematics III, 2003.

[29] D. Morris and T. Kanade. Image-consistent surface triangulation. In CVPR'00.

[30] E. Mouragnon, M. Lhuillier, M. Dhome, F. Dekeyser and P. Sayd. Generic and Real-Time Structure-from-Motion using Local Bundle Adjustment. In Image and Vision Computing, 27(8), pp. 1178-1193, 2009.

[31] R. Newcombe and A. Davison. Live Dense Reconstruction with a Single Moving Camera. In CVPR'10. 
[32] Q. Pan, G. Reitmayr, and T. Drummond. ProFORMA: probabilistic feature-based on-line rapid model acquisition. In $B M V C^{\prime} 09$.

[33] J.S. Sereni. Lecture 1 on Graph and Counting. INRIA, 2009.

[34] J.R. Shewchuk. Stabbing Delaunay Tetrahedralizations. Discrete and Computational Geometry, 32(3):339-343, 2004.

[35] G. Taubin. A signal processing approach to fair surface design. In SIGGRAPH'95.

[36] C. Taylor. Surface reconstruction from feature based stereo. In $I C C V^{\prime} 03$.

[37] Z. Wood, H. Hoppe, M. Desbrun and P. Schroder. Removing excess topology from isosurfaces. ACM Transactions on Graphics, 23(2), pp. 190-208, 2004.

[38] S. Yu and M. Lhuillier. Surface reconstruction of scenes using a catadiopric camera. In Mirage'11. Also in LNCS 6930.

[39] S. Yu and M. Lhuillier. Genus refinement of manifold surface reconstructed by sculpting the 3D-Delaunay triangulation of Structure-fromMotion points. In ICPR'12.

[40] S. Yu and M. Lhuillier. Incremental Reconstruction of Manifold Surface from Sparse Visual Mapping. In 3DIMPVT'12.

[41] H.K. Zhao, S. Osher and R. Fedkiw. Fast surface reconstruction using the level set method. In Workshop on Variational and Level Set Methods in Computer Vision, 2001.

[42] Q. Zhou, T. Ju and S. Hu. Topology repair of solid models using skeletons. IEEE Transactions on Visualization and Computer Graphics, 13(4), pp. 675-685, 2007. 